Provided for non-commercial research and education use. Not for reproduction, distribution or commercial use.

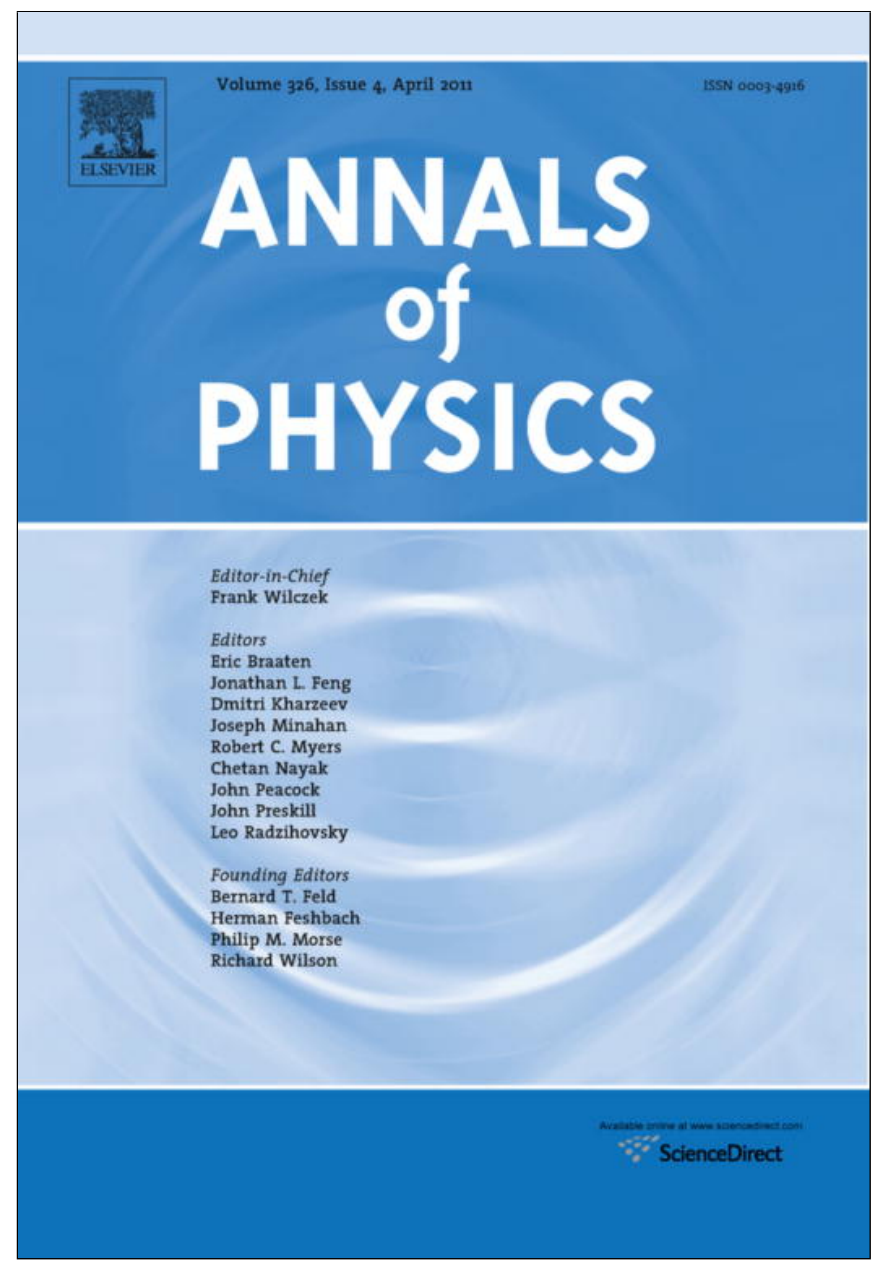

This article appeared in a journal published by Elsevier. The attached copy is furnished to the author for internal non-commercial research and education use, including for instruction at the authors institution and sharing with colleagues.

Other uses, including reproduction and distribution, or selling or licensing copies, or posting to personal, institutional or third party websites are prohibited.

In most cases authors are permitted to post their version of the article (e.g. in Word or Tex form) to their personal website or institutional repository. Authors requiring further information regarding Elsevier's archiving and manuscript policies are encouraged to visit:

http://www.elsevier.com/copyright 


\title{
Quantum phase transitions in Bose-Fermi systems
}

\author{
D. Petrellis ${ }^{\text {a }}$, A. Leviatan ${ }^{\mathrm{b}, *}, \mathrm{~F}$. Iachello ${ }^{\mathrm{a}}$ \\ ${ }^{a}$ Center for Theoretical Physics, Sloane Physics Laboratory, Yale University, New Haven, CT 06520-8120, USA \\ ${ }^{\mathrm{b}}$ Racah Institute of Physics, The Hebrew University, Jerusalem 91904, Israel
}

\section{A R T I C L E I N F O}

\section{Article history:}

Received 14 September 2010

Accepted 15 December 2010

Available online 23 December 2010

\section{Keywords:}

Bose-Fermi systems

Algebraic models

Quantum shape-phase transitions

Interacting boson-fermion model (IBFM)

\begin{abstract}
A B S T R A C T
Quantum phase transitions in a system of $N$ bosons with angular momentum $L=0,2(s, d)$ and a single fermion with angular momentum $j$ are investigated both classically and quantum mechanically. It is shown that the presence of the odd fermion strongly influences the location and nature of the phase transition, especially the critical value of the control parameter at which the phase transition occurs. Experimental evidence for the U(5)$\mathrm{SU}(3)$ (spherical to axially-deformed) transition in odd-even nuclei is presented.
\end{abstract}

(c) 2010 Elsevier Inc. All rights reserved.

\section{Introduction}

Quantum phase transitions (QPT) are qualitative changes in the structure of a physical system induced by a change in one or more parameters that appear in the quantum Hamiltonian describing the system. Originally introduced in nuclear physics [1,2], where they were called ground state phase transitions, they have received recently considerable attention in condensed matter physics and other areas [3]. Quantum phase transitions were investigated both classically [4,5] and quantum mechanically [6] in even-even nuclei in the early 1980's within the framework of the Interacting Boson Model (IBM), a model of nuclei in terms of correlated pairs of nucleons with $L=0,2$ treated as bosons ( $s, d$ bosons) [7]. In recent years, this study has been greatly expanded, including the aspect of symmetries (critical point symmetries [8-10], quasidynamical [11] and partial dynamical symmetries [12]) and their empirical evidence $[13,14]$, the study of finite- $N$ effects $[15-18]$ and of the corresponding scaling behavior [19-22]. The latter plays an important role in nuclei, since the nucleus is composed of a finite number of particles, $N$, and phase transitions, i.e. discontinuities in some quantities, are defined only in the limit $N \rightarrow \infty$. Also, the concept of QPTs has been enlarged to include excited states quantum phase

\footnotetext{
* Corresponding author. Tel.: +972 2 6585136; fax: +972 25611519.

E-mail addresses: dimitris.petrellis@yale.edu (D. Petrellis), ami@phys.huji.ac.il (A. Leviatan), francesco.iachello@yale.edu (F. Iachello).
} 
transitions (ESQPT) [23], that is qualitative changes in the structure of a physical system as a function of excitation energy. Quantum phase transitions in the IBM are particularly interesting since this model has an algebraic structure, $\mathrm{U}(6)$, which is rather complex, giving rise within its parameter space to both first and second order transitions. On the other side, because of its algebraic structure, one can work out the "phases" in explicit analytic form, since these correspond to dynamic symmetries of the Hamiltonian. This situation is summarized in several review papers [24-26], where a complete list of references is given.

In this article we address the problem of how QPTs are affected by the presence of fermions in addition to bosons (QPTs in Bose-Fermi systems, odd-even nuclei). Nuclei offer a unique opportunity to study this problem since one has a model, the Interacting Boson-Fermion Model (IBFM) [27], in terms of correlated pairs with $L=0,2$ ( $s, d$ bosons) and unpaired particles with angular momentum $j(j$ fermions), where this problem can be addressed in explicit form. (The problem is also of interest to the condensed matter physics community where one is interested in the motion of fermionic impurities in a bath of bosons [28].) Studies of QPTs in odd-even nuclei were implicitly initiated years ago by Scholten and Blasi [29]. Several explicit studies have recently been made by Alonso et al. [30-32] and by Böyükata et al. [33], who also have suggested a simple form of the IBFM Hamiltonian particularly well suited to study QPTs in odd-even nuclei because of its supersymmetric properties. Here we further expand on these studies and present the general theory of a single fermion with angular momentum $j$ interacting with a system of $s, d$ bosons (pairs with angular momentum $L=0,2$ ) and study QPTs in this system both classically and quantum mechanically. We consider specifically the case of a particle with $j=11 / 2$. After introducing the model Hamiltonian in Section 2, we present in Section 3 a classical analysis with novel results on single particle levels in a deformed field with both $\beta$ and $\gamma$ deformation of interest not only to the algebraic description but also to its geometric counterpart. In Section 4 we present a quantal analysis of the same problem, introduce correlation diagrams for Bose-Fermi systems and study the classical-quantal correspondence.

QPTs in odd-even nuclei would be a mere academic (and very complex) exercise were not for the fact that there is experimental evidence for the occurrence of these phase transitions in the odd-proton nuclei ${ }_{61} \mathrm{Pm},{ }_{63} \mathrm{Eu}$ and ${ }_{65} \mathrm{~Tb}$. In the last section (Section 5), we discuss this evidence and compare the data with realistic IBFM calculations. The evidence is particularly clear in the unique parity negative parity levels originating from the $h_{11 / 2}$ level $(\ell=5, j=11 / 2)$ and we therefore study these states. The large value of $j$ has also the advantage that it provides a way to unravel the many complications of QPTs in Bose-Fermi systems which are not present for small values of $j, j=1 / 2$ being trivial and $j=3 / 2$ being analytically solvable.

\section{Model Hamiltonian}

We consider here the model Hamiltonian of a system of $N$ bosons with angular momentum $L=0,2$ ( $s, d$ bosons) coupled to a fermion with angular momentum, $j$, as exemplified in the IBFM [27],

$$
H=H_{B}+H_{F}+V_{B F}
$$

with

$$
\begin{aligned}
& H_{B}=\varepsilon_{0}\left[(1-\xi) \hat{n}_{d}-\frac{\xi}{4 N} \widehat{Q}^{\chi} \cdot \widehat{Q}^{\chi}\right], \\
& H_{F}=\varepsilon_{j} \hat{n}_{j}, \\
& V_{B F}=V_{B F}^{M O N}+V_{B F}^{Q U A D}+V_{B F}^{E X C} .
\end{aligned}
$$

Here the subscripts $B, F$ and $B F$ refer to the boson, fermion, and boson-fermion parts of the full Hamiltonian and

$$
\begin{aligned}
& V_{B F}^{M O N}=A \hat{n}_{d} \hat{n}_{j}, \\
& V_{B F}^{Q U A D}=\Gamma \widehat{Q}^{\chi} \cdot \hat{q}_{j}, \\
& V_{B F}^{E X C}=\Lambda \sqrt{2 j+1}:\left[\left(d^{\dagger} \times \tilde{a}_{j}\right)^{(j)} \times\left(\tilde{d} \times a_{j}^{\dagger}\right)^{(j)}\right]^{(0)}: .
\end{aligned}
$$


The superscripts MON, QUAD and EXC label the monopole, quadrupole and exchange terms, respectively. Their coefficients are $A \equiv-A_{j} / \sqrt{5(2 j+1)}, \Gamma \equiv \Gamma_{j j} / \sqrt{5}$, and $\Lambda \equiv \Lambda_{j j}^{j} / \sqrt{2 j+1}$ in the notation of [27], and

$$
\begin{aligned}
& \hat{n}_{d}=d^{\dagger} \cdot \tilde{d}, \\
& \widehat{Q}^{\chi}=\left(d^{\dagger} \times s+s^{\dagger} \times \tilde{d}\right)^{(2)}+\chi\left(d^{\dagger} \times \tilde{d}\right)^{(2)}, \\
& \hat{n}_{j}=-\sqrt{2 j+1}\left(a_{j}^{\dagger} \times \tilde{a}_{j}\right)^{(0)}, \\
& \hat{q}_{j}=\left(a_{j}^{\dagger} \times \tilde{a}_{j}\right)^{(2)} .
\end{aligned}
$$

In these formulas, dots - denote scalar products, symbols $\times$ denote tensor products and : denotes normal ordering. Also, $s^{\dagger}, d_{\mu}^{\dagger}\left(s, d_{\mu}\right)(\mu=0, \pm 1, \pm 2)$ denote creation (annihilation) operators for $s, d$ bosons and $a_{j, m}^{\dagger}\left(a_{j, m}\right)\left(m= \pm \frac{1}{2}, \pm \frac{3}{2}, \ldots, \pm j\right)$ creation (annihilation) operators for fermions with angular momentum $j$. The adjoint operators are $\tilde{d}_{\mu}=(-)^{\mu} d_{-\mu}, \tilde{a}_{j, m}=(-)^{j-m} a_{j,-m}$.

The quantum phase transitions of the boson part of the Hamiltonian (2) are very well known [34]. There are three "phases", characterized by their symmetry, $\mathrm{U}(5), \mathrm{SU}(3)$ and $\mathrm{SO}(6)$, and two control parameters, $\xi$ and $\chi$. We consider here the range of parameters, $0 \leqslant \xi \leqslant 1,-\frac{\sqrt{7}}{2} \leqslant \chi \leqslant 0$. As $\xi$ changes from 0 to 1 the system undergoes a quantum phase transition. No phase transition occurs when $\chi$ changes from 0 to $-\frac{\sqrt{7}}{2}$. The phase transition as a function of $\xi$ is first order from $\mathrm{U}(5)$ to $\mathrm{SU}(3)$ (spherical to axially deformed phases) and second order from $\mathrm{U}(5)$ to $\mathrm{SO}(6)$ (spherical to $\gamma$-unstable deformed phases). The situation is summarized in Fig. 1, shown here for sake of later discussion.

In this paper we are interested in what happens when we vary the control parameters in the BoseFermi coupling, $A, \Gamma, \Lambda$, of Eq. (3). This problem is similar to (but more complex than) that of a bosonic system in an external field already discussed years ago by Landau and Lifshitz [35] where one is interested in what happens as one varies the strength of the field, and to that of a fermion in a bath of harmonic oscillator bosons recently discussed in condensed matter physics [28].

\section{Classical analysis}

Since the system we are considering here is that of an ensemble of bosons coupled to a single fermion, it is convenient to analyze the situation in terms of the motion of the single particle in the external field generated by the bosons [27]. This is done by introducing a (number projected) boson condensate and evaluating the expectation value of the Hamiltonian $H$ in the condensate [36,37]. This produces the fermion single particle Hamiltonian in the boson field, $\mathcal{H}$. Diagonalization of $\mathcal{H}$ gives the

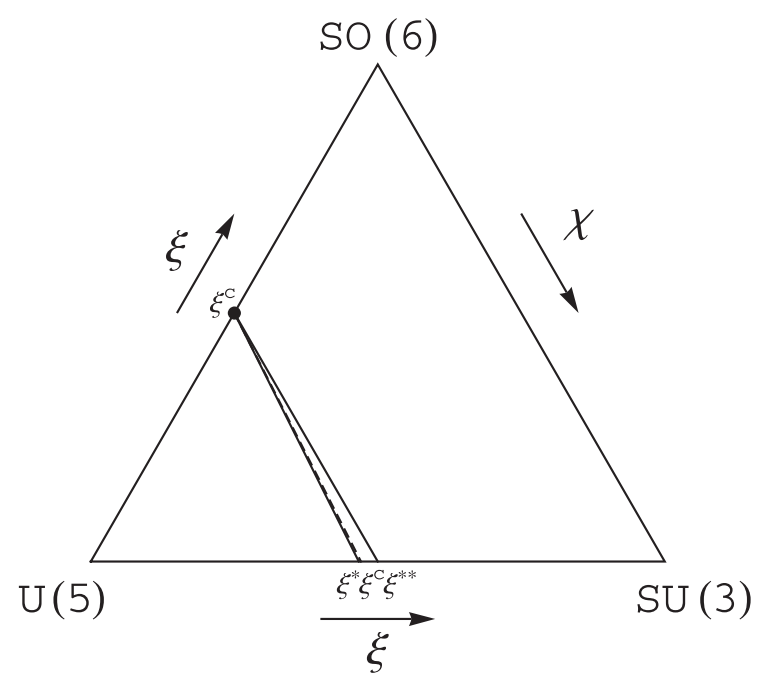

Fig. 1. Phase diagram of a system of $s, d$ bosons. The three phases are denoted by their symmetry, $U(5), S U(3)$ and $S O(6)$. A line of first order transitions ends in a point of second order transition in-between the $U(5)$ and $\mathrm{SO}(6)$ limits. The spinodal, critical and antispinodal points are denoted by $\xi^{*}, \xi^{c}, \xi^{* *}$, respectively. 
single particle energies. The total energy is the sum of the single particle energy and of the boson energy. Minimization of this total energy with respect to the boson classical variables gives the equilibrium values (classical order parameters). The behavior of the order parameter(s) as a function of the control parameter(s) determines the nature and order of the phase transition.

\subsection{Expectation value of $H_{B}$ in the boson condensate}

We begin by considering the boson condensate with good particle number $N[4,38,39]$

$$
|N ; \beta, \gamma\rangle=\frac{1}{\sqrt{N !}}\left[b_{c}^{\dagger}(\beta, \gamma)\right]^{N}|0\rangle
$$

with

$$
b_{c}^{\dagger}(\beta, \gamma)=\frac{1}{\left(1+\beta^{2}\right)^{1 / 2}}\left[\beta \cos \gamma d_{0}^{\dagger}+\frac{1}{\sqrt{2}} \beta \sin \gamma\left(d_{2}^{\dagger}+d_{-2}^{\dagger}\right)+s^{\dagger}\right] .
$$

The expectation value of the boson Hamiltonian $H_{B}$ of Eq. (2) in this condensate is given by [16]

$$
\begin{aligned}
E_{B}(N ; \beta, \gamma)= & \left\langle N ; \beta, \gamma\left|H_{B}\right| N ; \beta, \gamma\right\rangle \\
= & \varepsilon_{0} N\left\{\left(\frac{\beta^{2}}{1+\beta^{2}}\right)\left[(1-\xi)-\left(\chi^{2}+1\right) \frac{\xi}{4 N}\right]\right. \\
& \left.-\frac{5 \xi}{4 N\left(1+\beta^{2}\right)}-\frac{\xi}{4\left(1+\beta^{2}\right)^{2}} \frac{N-1}{N}\left[4 \beta^{2}-4 \sqrt{\frac{2}{7}} \chi \beta^{3} \cos 3 \gamma+\frac{2}{7} \chi^{2} \beta^{4}\right]\right\} .
\end{aligned}
$$

The limit $N \rightarrow \infty$ of this expectation value is of interest and it is

$$
\begin{aligned}
\bar{E}_{B}(\beta, \gamma) & =\lim _{N \rightarrow \infty} E_{B}(N ; \beta, \gamma) \\
& =\varepsilon_{0} N\left\{\left(\frac{\beta^{2}}{1+\beta^{2}}\right)(1-\xi)-\frac{\xi}{4} \frac{1}{\left(1+\beta^{2}\right)^{2}}\left[4 \beta^{2}-4 \sqrt{\frac{2}{7}} \chi \beta^{3} \cos 3 \gamma+\frac{2}{7} \chi^{2} \beta^{4}\right]\right\} .
\end{aligned}
$$

\subsection{Expectation value of $H_{F}$ and $V_{B F}$ in the boson condensate}

By integrating out the boson degrees of freedom, i.e. by taking the expectation value of $H_{F}$ and $V_{B F}$ in the boson condensate, one obtains the fermion Hamiltonian

$$
\mathcal{H}(N ; \beta, \gamma)=E_{B}(N ; \beta, \gamma)+\sum_{m_{1}, m_{2}}\left[\varepsilon_{j} \delta_{m_{1}, m_{2}}+g_{m_{1}, m_{2}}(N ; \beta, \gamma)\right]\left(\frac{a_{j, m_{1}}^{\dagger} a_{j, m_{2}}+a_{j, m_{2}}^{\dagger} a_{j, m_{1}}}{1+\delta_{m_{1}, m_{2}}}\right) .
$$

The matrix $g_{m_{1}, m_{2}}$ is a real, symmetric matrix, with explicit form given by

$$
g_{m_{1}, m_{2}}(N ; \beta, \gamma)=g_{m_{1}, m_{2}}^{M O N}(N ; \beta, \gamma)+g_{m_{1}, m_{2}}^{\mathrm{QUAD}}(N ; \beta, \gamma)+g_{m_{1}, m_{2}}^{E X X}(N ; \beta, \gamma)
$$

and

$$
\begin{aligned}
g_{m_{1}, m_{2}}^{\text {MON }}= & N A\left(\frac{\beta^{2}}{1+\beta^{2}}\right) \delta_{m_{1}, m_{2}} \equiv N A \tilde{g}_{m_{1}, m_{2}}^{\text {MON }}, \\
g_{m_{1}, m_{2}}^{\mathrm{QUAD}}= & N \Gamma\left(\frac{\beta}{1+\beta^{2}}\right)(-)^{j+m_{2}} \\
& \times\left\{\left[2 \cos \gamma-\chi \sqrt{\frac{2}{7}} \beta \cos 2 \gamma\right]\left\langle j, m_{1} ; j,-m_{2} \mid 2,0\right\rangle \delta_{m_{1}, m_{2}}\right. \\
& \left.+\left[\sqrt{2} \sin \gamma+\chi \sqrt{\frac{1}{7}} \beta \sin 2 \gamma\right]\left\langle j, m_{1} ; j,-m_{2} \mid 2,2\right\rangle\right\} \\
\equiv & -N \Gamma \tilde{g}_{m_{1}, m_{2}}^{\text {QUAD }},
\end{aligned}
$$




$$
\begin{aligned}
g_{m_{1}, m_{2}}^{E X C}= & N \Lambda\left(\frac{\beta^{2}}{1+\beta^{2}}\right)\left\{\left[\frac{X_{0}}{\sqrt{5(2 j+1)}}\right.\right. \\
& +(-)^{j+m_{2}}\left(\sqrt{\frac{2}{7}} X_{2} \cos 2 \gamma\left\langle j, m_{1} ; j,-m_{2} \mid 2,0\right\rangle\right. \\
& \left.\left.-\frac{1}{\sqrt{280}} X_{4}(7+5 \cos 2 \gamma)\left\langle j, m_{1} ; j,-m_{2} \mid 4,0\right\rangle\right)\right] \delta_{m_{1}, m_{2}} \\
& +(-)^{j+m_{2}}\left[-\sqrt{\frac{1}{7}} X_{2} \sin 2 \gamma\left\langle j, m_{1} ; j,-m_{2} \mid 2,2\right\rangle\right. \\
& \left.\left.-\sqrt{\frac{3}{28}} X_{4} \sin 2 \gamma\left\langle j, m_{1} ; j,-m_{2} \mid 4,2\right\rangle-\frac{1}{4}(1-\cos 2 \gamma) X_{4}\left\langle j, m_{1} ; j,-m_{2} \mid 4,4\right\rangle\right]\right\} \\
\equiv & N \Lambda \tilde{g}_{m_{1}, m_{2}}^{E X C} .
\end{aligned}
$$

Here $m_{1} \geqslant m_{2}$ and

$$
X_{L}=(2 j+1)\left\{\begin{array}{lll}
j & 2 & j \\
L & j & 2
\end{array}\right\}
$$

(The values for $m_{1}<m_{2}$ can be obtained by noting that the matrix is symmetric. The symbol $\langle\cdots \mid \ldots\rangle$ in Eqs. (12) and (13) denotes Clebsch-Gordan coefficients and the curly bracket in (14) is a 6j-symbol.) Also, in Eqs. (11)-(13) we have isolated the dependence on $N$ and on the parameters $A, \Gamma, \Lambda$, from that on the intrinsic variables $\beta$ and $\gamma$ by introducing the matrices $\tilde{g}^{M O N}, \tilde{g}^{Q U A D}, \tilde{g}^{E X C}$. The analysis of this section becomes then independent of the values of the couplings parameters and of $N$.

\subsection{Diagonalization of the $\tilde{g}$-matrix}

The basis for the diagonalization of $\tilde{g}$ is the fermion single-particle basis

$$
|j, m\rangle=a_{j, m}^{\dagger}|0\rangle \quad m= \pm j, \pm(j-1), \ldots, \pm \frac{1}{2} .
$$

Because of the structure of $\tilde{g}$, which couples basis states differing by \pm 2 in $m$, the diagonalization of $\tilde{g}$ splits into two (doubly degenerate) pieces with $m=j, j-2, j-4, \ldots,-(j-1)$ and similarly with $m \rightarrow-m$. The dimension of the basis is thus $j+\frac{1}{2}$. (In the case we discuss here, $j=11 / 2$, the matrices are $6 \times 6$. When $j=3 / 2$ the matrices are $2 \times 2$ and the results of the diagonalization can be obtained in explicit analytic form. When $j=1 / 2$, the matrix is $1 \times 1$ and only $\tilde{g}^{M O N}$ and the $X_{0}$ term in $\tilde{g}^{E X C}$ contribute trivially).

The diagonalization of the matrix $\tilde{g}_{m_{1}, m_{2}}(\beta, \gamma)$ yields the single particle eigenvalues

$$
\varepsilon_{i}(\beta, \gamma ; \chi) \quad i=1,2, \ldots, j+\frac{1}{2}
$$

and eigenfunctions

$$
\left|\psi_{i}(\beta, \gamma ; \chi)\right\rangle=\sum_{m} c_{m}^{i}(\beta, \gamma ; \chi)|j, m\rangle .
$$

These are the single particle levels in the deformed $\beta$ and $\gamma$ field generated by the bosons. They depend on the boson parameter $\chi$ which distinguishes different types of boson fields ( $\chi=0, \mathrm{SO}(6)$ symmetry; $\chi=-\frac{\sqrt{7}}{2}, \mathrm{SU}(3)$ symmetry).

The contribution to the classical energy coming from the Bose-Fermi interaction is the single particle energy

$$
e_{i}(N ; \beta, \gamma ; \chi ; A, \Gamma, \Lambda)=N\left[A \varepsilon_{i}^{M O N}(\beta, \gamma ; \chi)-\Gamma \varepsilon_{i}^{\mathrm{QUAD}}(\beta, \gamma ; \chi)+\Lambda \varepsilon_{i}^{E X C}(\beta, \gamma ; \chi)\right] .
$$

This contribution is proportional to the number of bosons $N$ as it should since the Bose-Fermi interaction, $V_{B F}$, is linear in the generators of $\mathrm{U}(6)$. 


\subsubsection{Results for the single particle eigenvalues}

(a) The monopole term. This term is diagonal with eigenvalues which do not depend on the index $i$ and on the parameter $\chi$

$\varepsilon_{i}^{\mathrm{MON}}(\beta, \gamma ; \chi)=\left(\frac{\beta^{2}}{1+\beta^{2}}\right)$.

(b) The quadrupole term. The eigenvalues of the matrix $\tilde{g}_{m_{1}, m_{2}}^{\text {QUAD }}$ must be obtained numerically. A program has been written in Mathematica to diagonalize the matrix. Using this program, we have first investigated the symmetries of the eigenvalues. Fig. 2 shows the eigenvalues $\varepsilon_{i}^{\text {QUAD }}(\beta, \gamma ; \chi)$ as a function of $\gamma$ in the domain $0 \leqslant \gamma \leqslant 2 \pi$ for $\chi=-\frac{\sqrt{7}}{2}$ and $\beta=\sqrt{2}$ (top part) and for $\chi=0, \beta=1$ (bottom part). (We have chosen these values of $\beta$ because they are the equilibrium values, $\beta_{e, B}$, of the boson energy functional, $\bar{E}_{B}(\beta, \gamma)$, Eq. (8), for $\operatorname{SU}(3), \chi=-\frac{\sqrt{7}}{2}$ and $\left.\operatorname{SO}(6), \chi=0\right)$. The various states are labelled by the index $i=1, \ldots, 6$ in order of increasing energy. This figure shows the remarkable result that the single particle energies generated by the quadrupole Bose-Fermi interaction are periodic in $\gamma$ with period $\frac{2 \pi}{3}$ and are symmetric under reflections around $\frac{\pi}{3}$. This is in spite of the fact that $\tilde{g}$ contains terms $\cos \gamma, \sin \gamma, \cos 2 \gamma, \sin 2 \gamma$. We can therefore restrict our
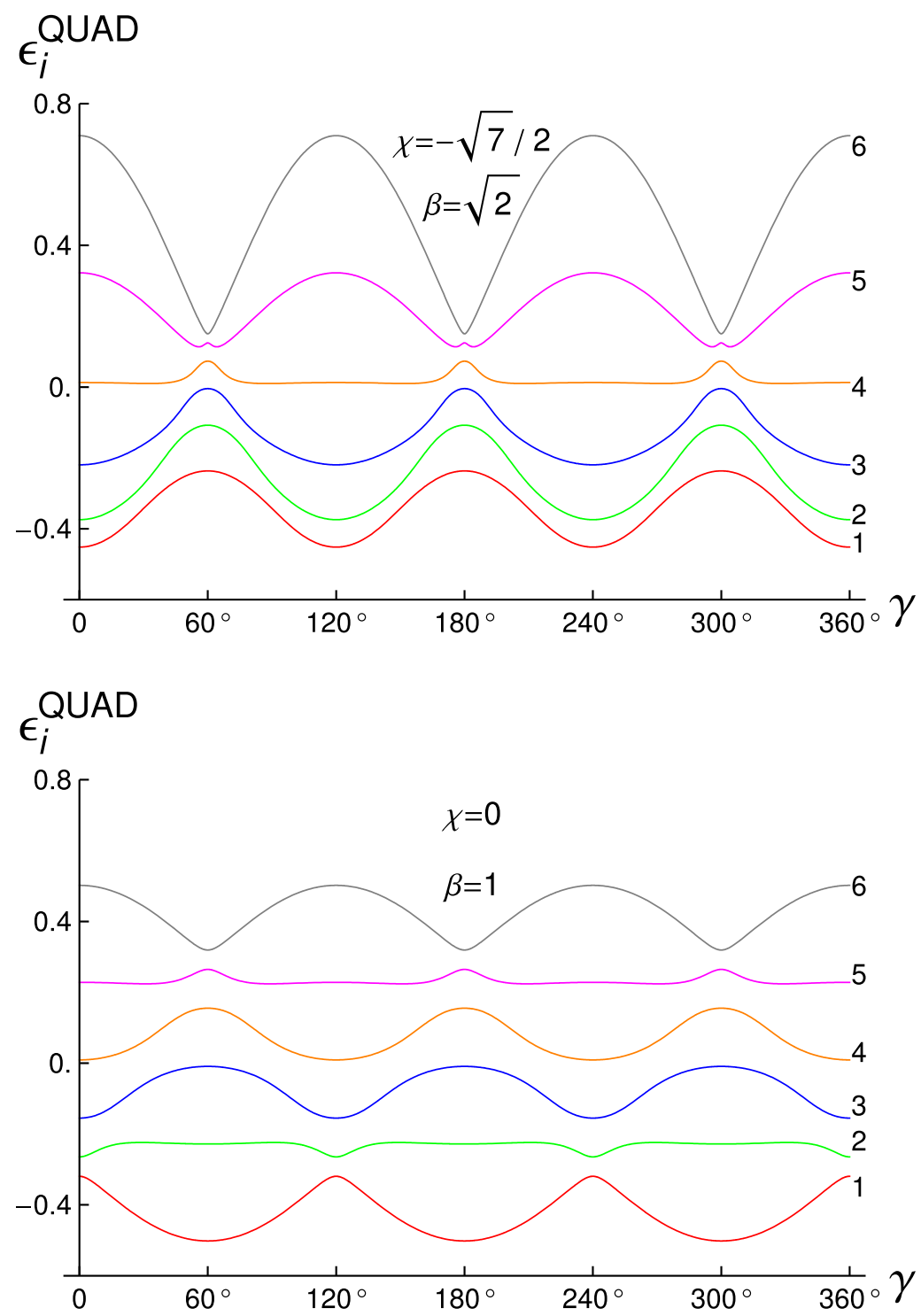

Fig. 2. Dependence on $\gamma$ of the eigenvalues $\varepsilon_{i}^{\text {QUAD }}(\beta, \gamma ; \chi)(i=1, \ldots, 6)$ of the matrix $\tilde{g}_{m_{1}, m_{2}}^{\text {QUAD }}$ (12) for $\chi=-\frac{\sqrt{7}}{2}, \beta=\sqrt{2}$ (top part) and $\chi=0, \beta=1$ (bottom part), labelled in order of increasing energy. 
study to $0 \leqslant \gamma \leqslant \frac{\pi}{3}$. (The periodicity $\frac{2 \pi}{3}$ can be proven by constructing the characteristic polynomial that diagonalizes the matrix and showing that it is a function of $\cos 3 \gamma$ ). From Fig. 2 one can see that the $\gamma$ dependence of the single particle levels is different for $\chi=-\frac{\sqrt{7}}{2}$ than for $\chi=0$. In Figs. 3 and 4 we show the eigenvalues $\varepsilon_{i}^{\text {QUAD }}(\beta, \gamma ; \chi)$ as a function of $\beta\left(\right.$ at $\left.\gamma=0^{\circ}\right)$, in the interval $-2 \leqslant \beta \leqslant 2$ (top part), and as a function of $\gamma$ in the interval $0^{\circ} \leqslant \gamma \leqslant 60^{\circ}$ at $\beta=\sqrt{2}$ for $\chi=-\frac{\sqrt{7}}{2}$ and at $\beta=1$ for $\chi=0$ (bottom part). The bottom part is identical to Fig. 2 but restricted to $0^{\circ} \leqslant \gamma \leqslant 60^{\circ}$. We see that when $\chi=0$ we have an additional symmetry

$\varepsilon_{i}^{Q U A D}(\beta, \gamma ; 0)=-\varepsilon_{i}^{Q U A D}(-\beta, \gamma ; 0)$.

This symmetry is lost when $\chi \neq 0$. By combining the symmetries in $\gamma$ with those in $\beta$ we find that the single particle energy eigenvalues $\varepsilon_{i}^{\mathrm{QUAD}}(\beta, \gamma ; \chi)$ are invariant, when $\chi=0$, under the transformation

$\varepsilon_{i}^{\mathrm{QUAD}}\left(-\beta, \gamma=60^{\circ} ; 0\right)=\varepsilon_{i}^{\mathrm{QUAD}}\left(\beta, \gamma=0^{\circ} ; 0\right)$.

This symmetry is lost when $\chi \neq 0$. In order to recover it, we need to simultaneously change the boson field from $\chi<0$ (prolate) to $\chi>0$ (oblate) when we go from $\beta>0$ to $\beta<0$. The single particle energies have then the property
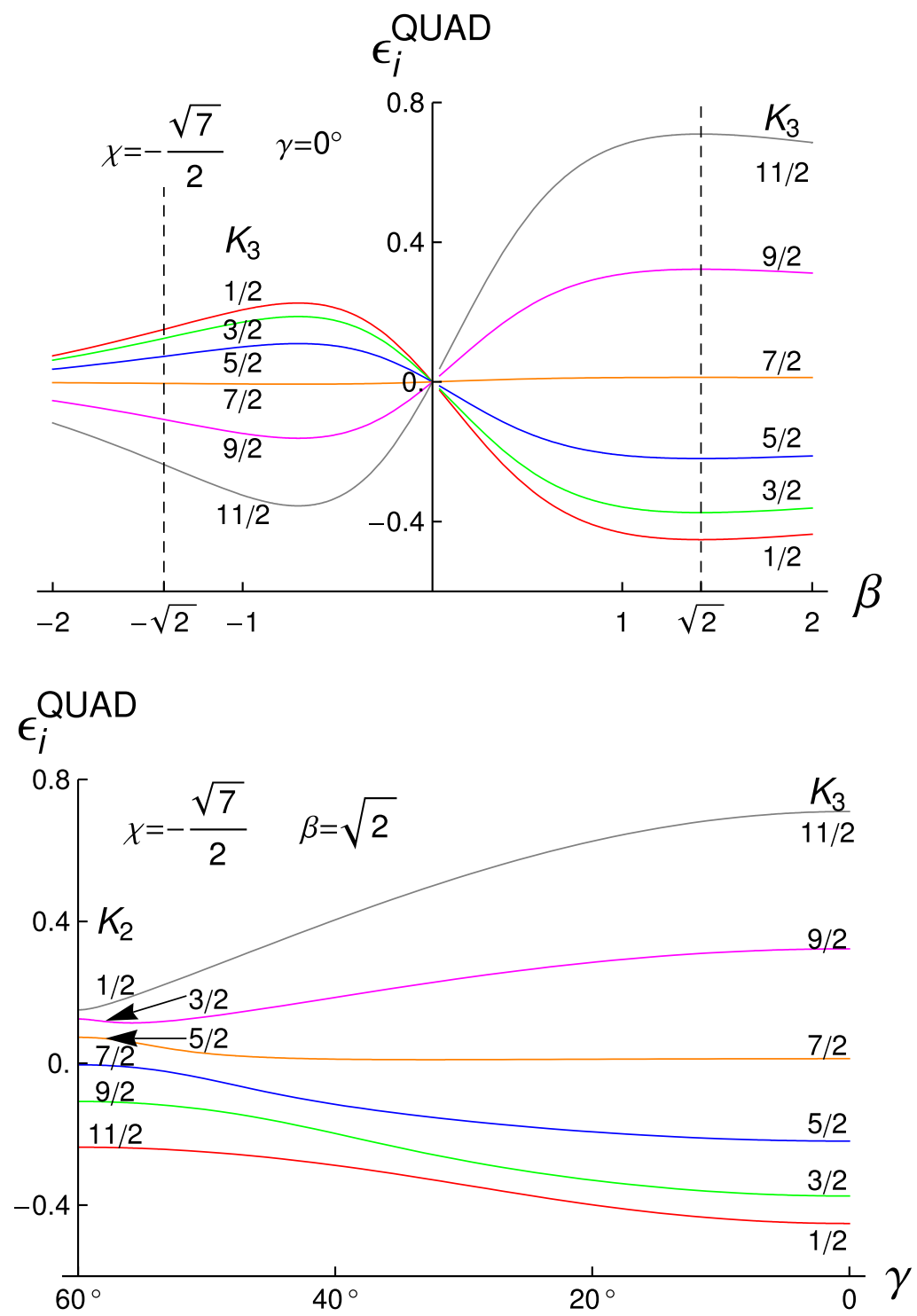

Fig. 3. Eigenvalues $\varepsilon_{i}^{Q U A D}(\beta, \gamma ; \chi)$ for a particle with angular momentum $j=11 / 2$ in a quadrupole $\beta, \gamma$ field with $\chi=-\frac{\sqrt{7}}{2}$ (prolate field) as a function of $\beta$ for $\gamma=0^{\circ}$ (top part) and as a function of $\gamma$ for $\beta=\sqrt{2}$ (bottom part). The eigenvalues are labelled by the projection of the angular momentum on the $\hat{3}$ axis at $\gamma=0^{\circ}$ and on the $\hat{2}$ axis at $\gamma=60^{\circ}$. 

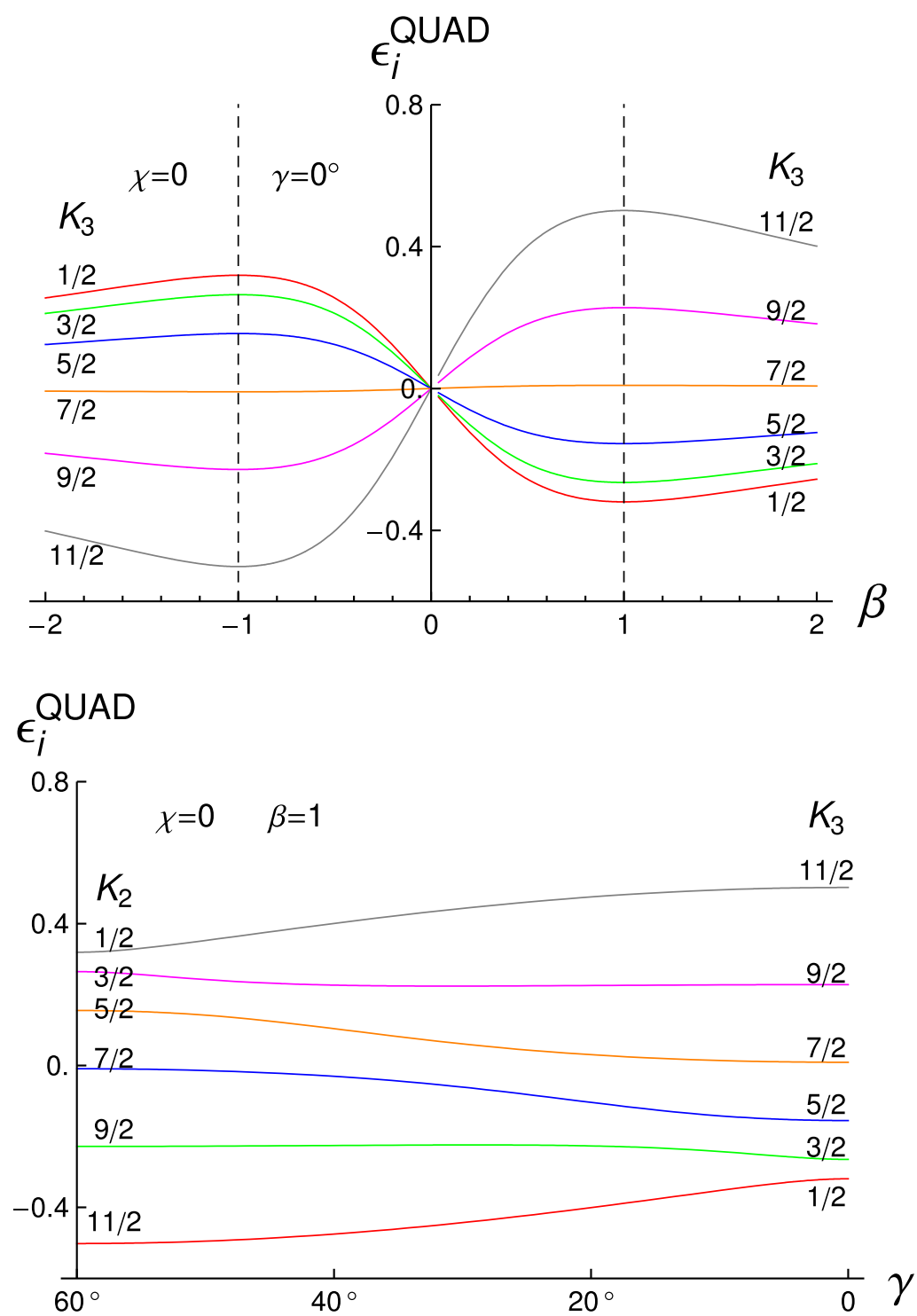

Fig. 4. Eigenvalues $\varepsilon_{i}^{\mathrm{QUAD}}(\beta, \gamma ; \chi)$ for a particle with angular momentum $j=11 / 2$ in a quadrupole $\beta, \gamma$ field with $\chi=0$ ( $\gamma$-unstable field) as function of $\beta$ for $\gamma=0^{\circ}$ (top part) and as a function of $\gamma$ for $\beta=1$ (bottom part). Labelling as in Fig. 3.

$$
\varepsilon_{i}^{\mathrm{QUAD}}\left(-\beta, \gamma=60^{\circ} ;-\chi\right)=\varepsilon_{i}^{\mathrm{QUAD}}\left(\beta, \gamma=0^{\circ} ; \chi\right) \text {. }
$$

For the purpose of this article, where we are interested in the effect of a single fermion on a bosonic system with a definite value of $\chi \leqslant 0$ (prolate boson field) it is sufficient to restrict all further analysis to the domain $\beta \geqslant 0,0^{\circ} \leqslant \gamma \leqslant 60^{\circ}$.

We also note that the matrix $\tilde{g}_{m_{1}, m_{2}}^{\text {QUAD }}$ at $\gamma=0^{\circ}$ is diagonal. Its eigenvalues are given in terms of the projection $K_{3}$ of the angular momentum on the intrinsic axis $\hat{3}$. The eigenvalues at $\gamma=60^{\circ}$ are instead given in terms of the projection $K_{2}$ on the intrinsic axis $\hat{2}$. There is a continuous change from $K_{3}$ to $K_{2}$ with the correspondence shown in Figs. 3 and 4. The single particle levels in a $\beta, \gamma$ field were studied years ago by Meyer-ter-Vehn [40] within the framework of the collective model. The $\gamma$ dependence of our results for $\chi=0$ is identical to that of [40]. On the other side the $\beta$ dependence differs for large $\beta$ due to the denominators $\frac{1}{1+\beta^{2}}$ in our $\tilde{g}$-matrix and to the fact that the variable $\beta$ of the collective model, $\beta_{B M}$, is related to that of the Interacting Boson Model, $\beta_{I B M}$, by a scale transformation, $\beta_{B M}=c \beta_{I B M}$ with $c \approx 0.2$ in the mass $A=150$ region [7]. We also note that, if the variable $\gamma$ is frozen to $0^{\circ}$, one obtains the single particle levels in a deformed $\beta$ field with axial symmetry. Those were studied years ago by Nilsson within the framework of the collective model [41]. Our results for $\chi=0$ are similar to those of the Nilsson model except for the denominators $\frac{1}{1+\beta^{2}}$. 
(c) The exchange term. The eigenvalues of the matrix $\tilde{g}_{m_{1}, m_{2}}^{E X C}$ are also obtained numerically. We first investigate the symmetries of the eigenvalues. Fig. 5 shows the eigenvalues $\varepsilon_{i}^{\operatorname{EX}}(\beta, \gamma ; \chi)$ as a function of $\gamma$ in the domain $0 \leqslant \gamma \leqslant 2 \pi$ for $\chi=-\frac{\sqrt{7}}{2}, \beta=\sqrt{2}$ and $\chi=0, \beta=1$. This figure shows that the contributions to the single particle energies coming from the exchange interaction are periodic in $\gamma$ with period $\frac{\pi}{3}$, that is half of the period of the quadrupole interaction, and are symmetric under reflection around $\frac{\pi}{6}$, i.e. they are a function of $\cos 6 \gamma$. Furthermore, the exchange term is independent of $\chi$ and is an even function of $\beta$.

(d) Combination of quadrupole and exchange. We consider here the eigenvalues, $\varepsilon_{i}^{\mathrm{QUAD}+E X C}(\beta, \gamma ; \chi)$, of the matrix

$\tilde{g}_{m_{1}, m_{2}}^{Q U A D+E X C}(\beta, \gamma ; \chi)=-\tilde{g}_{m_{1}, m_{2}}^{Q U A D}(\beta, \gamma ; \chi)+\left(\frac{\Lambda}{\Gamma}\right) \tilde{g}_{m_{1}, m_{2}}^{E X C}(\beta, \gamma ; \chi)$

and, for sake of display, we take $\left(\frac{1}{T}\right)=3$. The eigenvalues of this matrix are shown in Figs. 6 and 7. We see from these figures that the ordering of the single-particle eigenvalues at a given $\beta, \gamma$ when both quadrupole and exchange interactions are present is rather complex. Our results are similar to those of the Nilsson model plus BCS [27]. The similarity is no longer so obvious as in
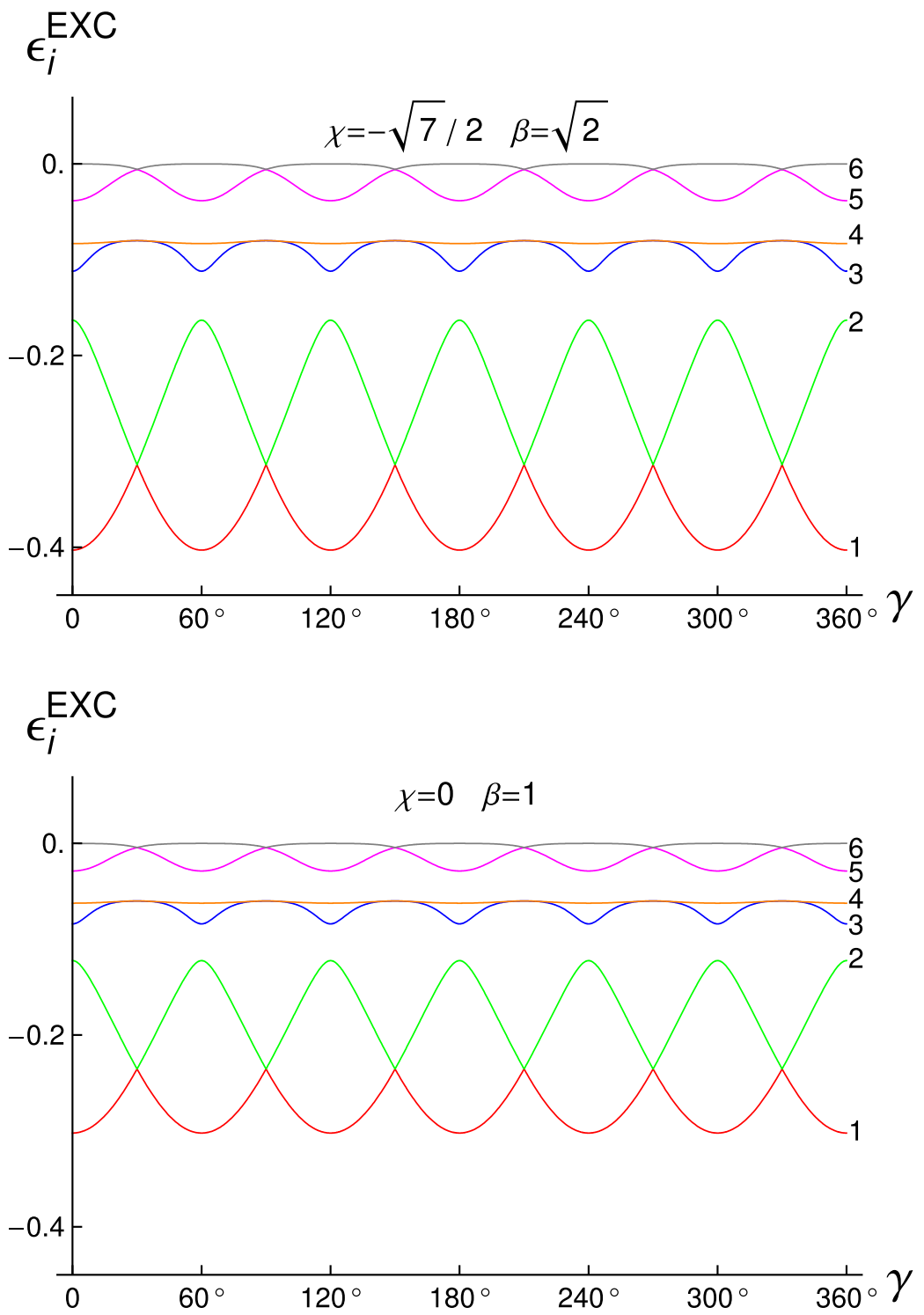

Fig. 5. Dependence on $\gamma$ of the eigenvalues $\varepsilon_{i}^{E X C}(\beta, \gamma ; \chi)(i=1, \ldots, 6)$ of the matrix $\tilde{g}_{m_{1}, m_{2}}^{E X C}$ for $\chi=-\frac{\sqrt{7}}{2}, \beta=\sqrt{2}$ (top part) and $\chi=0$, $\beta=1$ (bottom part), labelled in order of increasing energy. 
the case in which the coupling is purely quadrupole, but it can be seen by solving the BCS equations in the deformed $\beta, \gamma$ field as done by Meyer-ter-Vehn [40].

The formalism is flexible enough that one can also investigate particle-hole conjugation. Within the framework of IBFM, particle-hole conjugation is the transformation $\Gamma \rightarrow-\Gamma, \Lambda \rightarrow \Lambda$ (see the following Eq. (38)). When $\Lambda=0$, the effect of this transformation is to reverse the order of the single particle levels in the previous Figs. 3 and 4 , as one can simply see from the form of $g^{Q U A D}(\beta, \gamma ; \chi)$ in Eq. (12). When $\Lambda \neq 0$ the transformation leads to a different ordering of the single particle levels. We do not dwell on this point further, but note instead that a special circumstance occurs when the variable $\gamma$ is frozen to $0^{\circ}$ (prolate axial symmetry). As remarked above, the quadrupole Bose-Fermi interaction is diagonal at $\gamma=0^{\circ}$. It turns out that also the exchange interaction is diagonal. The eigenvalues of the $g$-matrix for the combined quadrupole plus exchange interaction are given by [36]

$$
\begin{aligned}
\lambda_{K}(\beta)= & -N \Gamma\left\{\left(\frac{\beta}{1+\beta^{2}}\right) \sqrt{5}\left(2-\beta \chi \sqrt{\frac{2}{7}}\right) P_{j}\left[3 K^{2}-j(j+1)\right]\right\} \\
& -N \Lambda\left\{\left(\frac{\beta^{2}}{1+\beta^{2}}\right)(2 j+1) P_{j}^{2}\left[3 K^{2}-j(j+1)\right]^{2}\right\},
\end{aligned}
$$

where
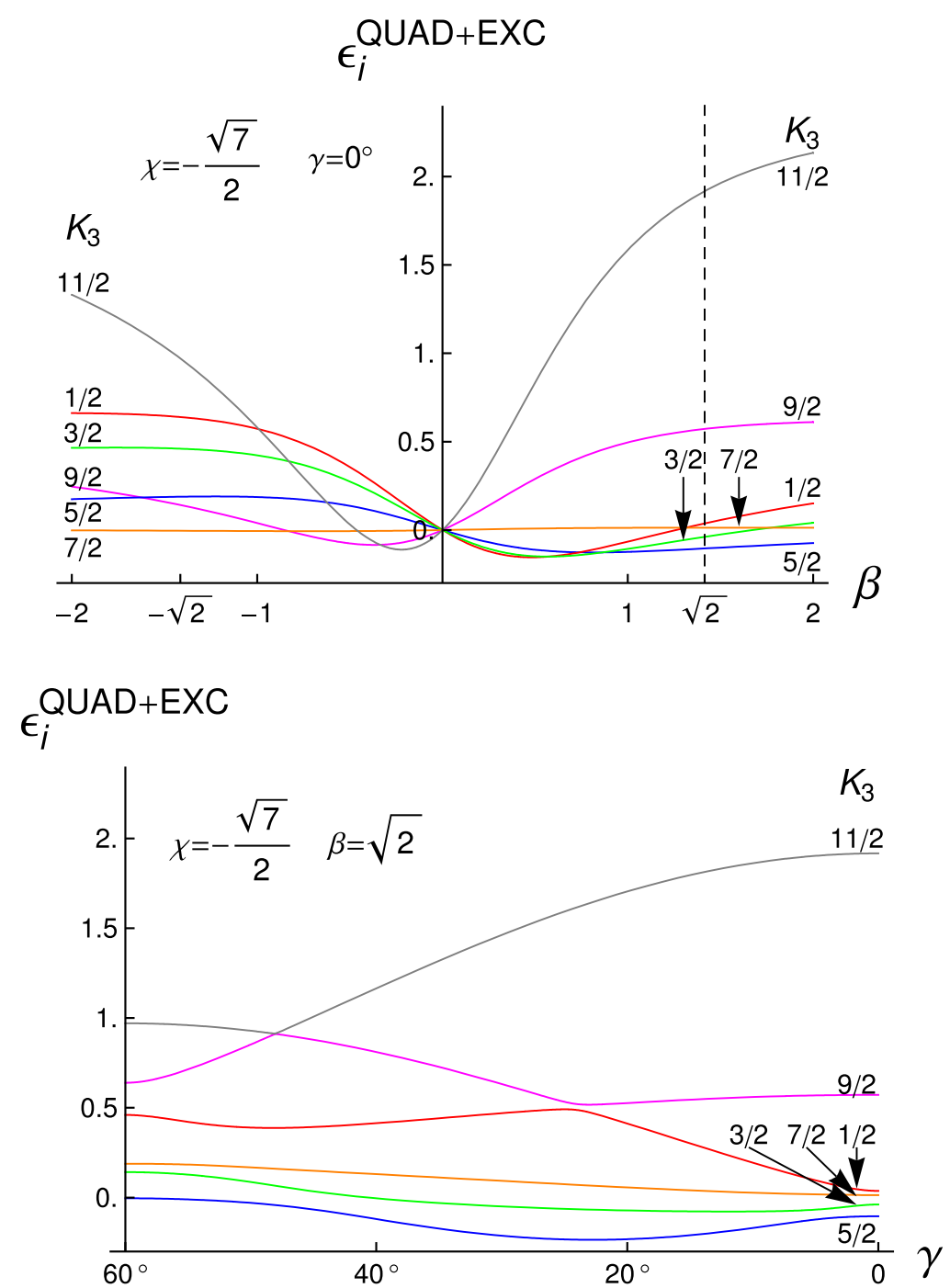

Fig. 6. Eigenvalues $\varepsilon_{i}^{\mathrm{QUAD}+\mathrm{EXC}}(\beta, \gamma ; \chi)$ for a particle with $j=11 / 2$ in an external $\beta, \gamma ; \chi=-\frac{\sqrt{7}}{2}$ field with both quadrupole and exchange interaction with strengths $\Lambda / \Gamma=3$. Labelling as in Fig. 3. Figure constructed by using $\Gamma=-0.0958, \Lambda=-0.287$. 

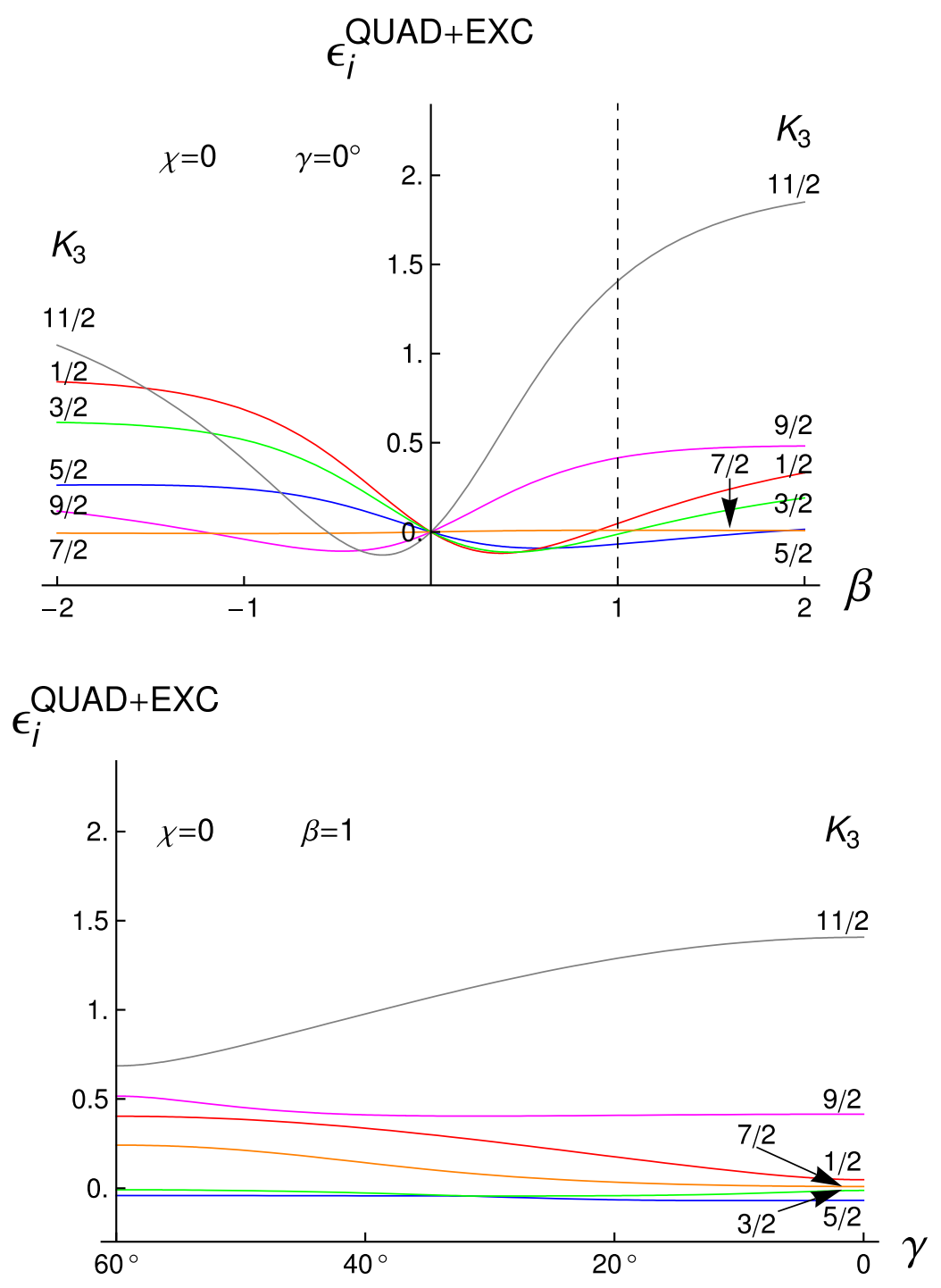

Fig. 7. Same as Fig. 6 but with $\chi=0$.

$$
P_{j}=[(2 j-1) j(2 j+1)(j+1)(2 j+3)]^{-1 / 2} .
$$

The eigenvalues can be labelled by the projection of the angular momentum on the intrinsic axis $\hat{3}, K_{3} \equiv K=\frac{1}{2}, \frac{3}{2}, \ldots, j$ and they are doubly degenerate.

The eigenvalues of the $g$-matrix for the combined quadrupole plus exchange interaction are also diagonal at $\gamma=60^{\circ}$ (oblate axial symmetry), and are given by

$$
\begin{aligned}
\omega_{K_{2}}(\beta)= & -N \Gamma\left\{\left(\frac{\beta}{1+\beta^{2}}\right) \sqrt{5}\left(-2-\beta \chi \sqrt{\frac{2}{7}}\right) P_{j}\left[3 K_{2}^{2}-j(j+1)\right]\right\} \\
& -N \Lambda\left\{\left(\frac{\beta^{2}}{1+\beta^{2}}\right)(2 j+1) P_{j}^{2}\left[3 K_{2}^{2}-j(j+1)\right]^{2}\right\},
\end{aligned}
$$

where $K_{2}$ is the projection of the angular momentum on the intrinsic axis $\hat{2}$. They satisfy the relation

$$
\omega_{K_{2}}(\beta)=\lambda_{K_{3} \rightarrow K_{2}}(-\beta) .
$$

\subsection{Equilibrium values: classical order parameters}

Having determined the single particle energies, $e_{i}$, Eq. (18), we can construct the total energy surface 


$$
E_{i}(N ; \beta, \gamma ; \xi, \chi ; A, \Gamma, \Lambda)=E_{B}(N ; \beta, \gamma ; \xi, \chi)+\varepsilon_{j}+e_{i}(N ; \beta, \gamma ; \chi ; A, \Gamma, \Lambda),
$$

where $E_{B}$, Eq. (7), and hence $E_{i}$ depend also on the scale parameter $\varepsilon_{0}$. In the traditional approach to odd-even nuclei, the equilibrium values $\beta_{e}, \gamma_{e}$ are obtained by minimizing the boson energy $E_{B}$ (or core energy in the collective model). This provides a static deformation and the single particle energies are evaluated in this static deformation. This is a good approximation when the deformation is large. In the transitional region, this is no longer the case. A better approximation is to minimize the total energy surface including the contribution of the fermions (the odd-particle). Although the fermion contribution is of order $1 / N$, it will be shown that it has a dramatic effect on the phase transition, modifying the location of the critical point.

The equilibrium values $\beta_{e}$ and $\gamma_{e}$ (the classical order parameters) for the combined system are obtained by minimizing $E_{i}$ with respect to $\beta$ and $\gamma$, i.e. imposing the conditions

$$
\frac{\partial E_{i}}{\partial \beta}=0, \quad \frac{\partial E_{i}}{\partial \gamma}=0
$$

Minimization of $E_{i}$ is, in general, no simple matter. The boson part, $E_{B}$, has a minimum at $\beta_{e, B}(\xi)$ and at $\gamma_{e, B}=0^{\circ}$, for any value of $\xi$ and $\chi<0$, except for $\chi=0$ where there is no minimum in $\gamma(\gamma$-unstable situation). The boson equilibrium values $\beta_{e, B}(\xi)$ have been calculated by several authors [16] and are shown in Fig. 8 as a function of the control parameter $\xi$ for two values of $\chi=-\frac{\sqrt{7}}{2}$ and $\chi=0$, for later comparison with those in odd-even nuclei (Bose-Fermi system).

The single particle eigenvalues $\varepsilon_{i}$ have a rather complex structure (Figs. 3 and 4), especially when there is an exchange interaction (Figs. 6 and 7). When doing the minimization, an important question is on what space to minimize. In view of the periodicity in $\gamma$, it is sufficient to minimize $E_{i}$ in the sex$\operatorname{tant} 0^{\circ} \leqslant \gamma \leqslant 60^{\circ}$. Also, since the purpose of this article is to understand the extent to which the presence of an odd fermion modifies the phase transition, we seek solutions in the neighbourhood of the boson equilibrium values and thus we do a constrained minimization with $\beta \geqslant 0$, in practice $0 \leqslant \beta \leqslant 2$.

To fully investigate QPTs in IBFM, one should do a study as a function of all control parameters $\xi, \chi$ (bosons) and $A, \Gamma, \Lambda$ (Bose-Fermi). In this paper we study two phase transitions: (i) $\mathrm{U}(5)$ to $\mathrm{SU}(3)$, spherical to axially deformed, $\chi=-\frac{\sqrt{7}}{2}$ and (ii) $\mathrm{U}(5)$ to $\mathrm{SO}(6)$, spherical to $\gamma$-unstable, $\chi=0$, both induced by a change in $0 \leqslant \xi \leqslant 1$. These phase transitions have been investigated extensively in the purely bosonic case and are of practical importance. We also set $A=0$, since the monopole term only renormalizes the $\hat{n}_{d}$ term in the boson Hamiltonian, $H_{B}$, and its effects can be easily seen. We are left with two additional control parameters, $\Gamma$ and $\Lambda$. In order to isolate the dependence on the fermion angular momentum $j$, we write

$$
\begin{aligned}
& \Gamma=\Gamma_{0} Q_{j j}, \\
& Q_{j j}=\left\langle j\left\|Y^{(2)}\right\| j\right\rangle=\sqrt{\frac{5}{4 \pi}} \sqrt{2 j+1}\langle j, 1 / 2 ; 2,0[j, 1 / 2\rangle,
\end{aligned}
$$
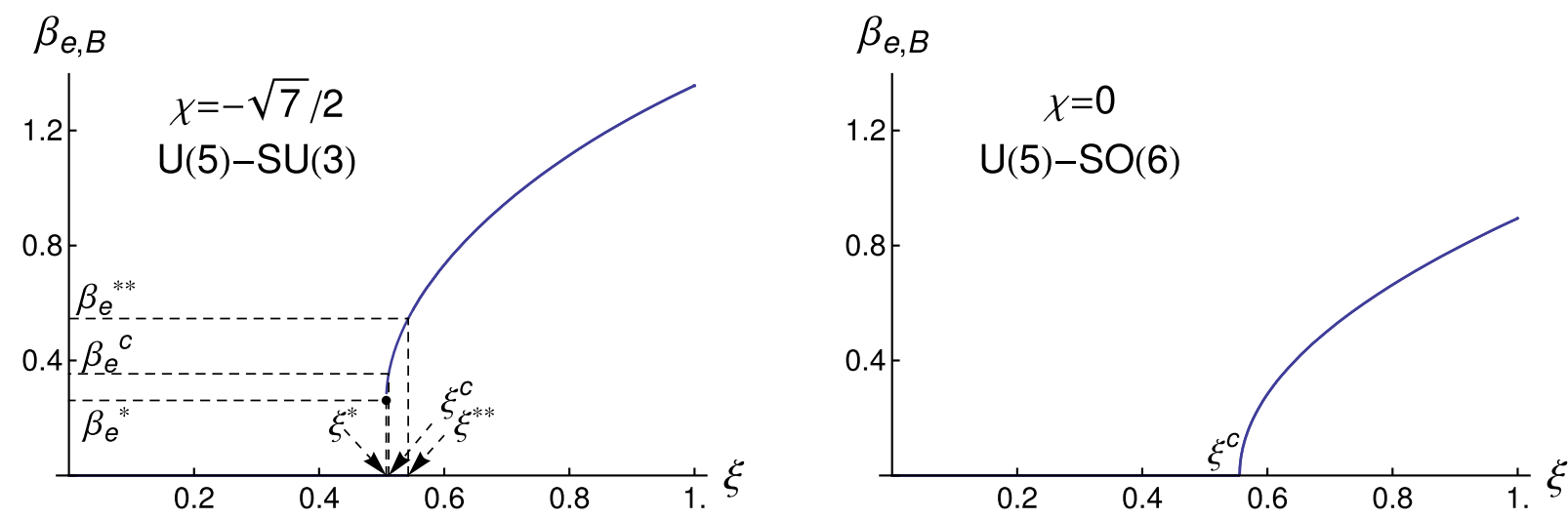

Fig. 8. Equilibrium values, $\beta_{e, B}$, as a function of the control parameter, $\xi$, of the bosonic part of the energy functional, $E_{B}$, for the transition $\mathrm{U}(5)-\mathrm{SU}(3), \chi=-\frac{\sqrt{7}}{2}$, left panel, and $\mathrm{U}(5)-\mathrm{SO}(6), \chi=0$, right panel. The spinodal, critical and antispinodal points are denoted by $\xi^{*}, \xi^{c}, \xi^{* *}$, respectively. Figure constructed for $N=10$ bosons. 
where the double-bar in Eq. (30) denotes a reduced matrix element. To further restrict the study, we take $\Gamma_{0}$ proportional to $\xi$

$$
\Gamma_{0}=-2 \varepsilon_{0} \frac{\xi}{4 N}
$$

The entire study presented here is thus in terms of a single control parameter $\xi$, plus the value of $\Lambda$ which, for convenience, can also be written as

$$
\Lambda=\lambda \varepsilon_{0}
$$

making all terms in $H$ then proportional to the scale factor $\varepsilon_{0}$. The parameterization of Eq. (31), for the quadrupole term, was suggested by Alonso et al. [30] and it has the advantage that the combined Hamiltonian can be rewritten, apart from an overall constant, as

$$
\begin{aligned}
H= & \varepsilon_{0}\left\{(1-\xi)\left(\hat{n}_{d}+\hat{n}_{j}\right)-\frac{\xi}{4 N}\left(\widehat{Q}^{\chi}+\hat{q}_{F}\right) \cdot\left(\widehat{Q}^{\chi}+\hat{q}_{F}\right)\right. \\
& \left.+\lambda \sqrt{2 j+1}:\left[\left(d^{\dagger} \times \tilde{a}_{j}\right)^{(j)} \times\left(\tilde{d} \times a_{j}^{\dagger}\right)^{(j)}\right]^{(0)}:\right\},
\end{aligned}
$$

where

$$
\hat{q}_{F}=Q_{j j} \hat{q}_{j}
$$

and $\hat{q}_{j}=\left(a_{j}^{\dagger} \times \tilde{a}_{j}\right)^{(2)}$. If one multiplies $Q_{j j}$ by $-\sqrt{\pi}$, the corresponding Hamiltonian for $j=3 / 2$ and $\chi$ $=\lambda=0$, has a Bose-Fermi symmetry $\mathrm{SO}(6) \otimes \mathrm{SU}(4) \supset \operatorname{Spin}(6)$ [27], i.e., the Hamiltonian is invariant under interchange of the boson operators $\hat{n}_{d}, \widehat{Q}^{\chi}$ with the fermion operators $\hat{n}_{j}, \hat{q}_{j}$.

\subsubsection{Results for $\Lambda=0$}

The equilibrium values for the $\mathrm{U}(5)-\mathrm{SU}(3)$ transition are shown in Fig. 9 and, for the $\mathrm{U}(5)-\mathrm{SO}(6)$ transition, in Fig. 10. From these figures one can see the effects of the odd particle on the phase transition. In the $\mathrm{U}(5)-\mathrm{SU}(3)$ case, the phase transition is washed out for states $1-3$ and enhanced for states 4-6. The critical point is approximately at the same location as for the purely bosonic case $\left(\xi_{B}^{c} \sim 0.51\right)$ for state $4\left(\xi_{4}^{c} \sim 0.50\right)$, but it is moved to larger values for state $5\left(\xi_{5}^{c} \sim 0.53\right)$ and state $6\left(\xi_{6}^{c} \sim 0.58\right)$. The values of $\gamma_{e}$ below and around the critical point are no longer zero. After the critical point, all states become those of a single particle in an axially deformed field $\left(\gamma_{e}=0^{\circ}\right)$ with equal deformation $\beta_{e, i}=\beta_{e, B}$. States in this region can be labelled by the projection $K=1 / 2,3 / 2,5 / 2,7 / 2,9 / 2,11 / 2$ of the angular momentum on the intrinsic $\hat{3}$ axis, corresponding to states $i=1, \ldots, 6$. A different situation occurs for the $\mathrm{U}(5)-\mathrm{SO}(6)$ transition. Although the equilibrium values, $\beta_{e, i}$, follow the same behavior as for the $\mathrm{U}(5)-\mathrm{SU}(3)$ transition, the equilibrium values $\gamma_{e, i}$ do not. States 1 and 6 are oblate $\left(\gamma_{e}=60^{\circ}\right)$, states $2-4$ are prolate $\left(\gamma_{e}=0^{\circ}\right)$ and state 5 is triaxial $\left(\gamma_{e}=30^{\circ}\right)$. For this phase transition, the equilibrium value of $\gamma$ is dictated by that of the single particle states, $e_{i}$, since the boson part is $\gamma$ independent. The effect of the odd-particle is more dramatic here than in the case of the $U(5)-S U(3)$ transition.

\subsubsection{Results for $\Lambda \neq 0$}

The equilibrium values when $\Lambda=-0.287$ for the $\mathrm{U}(5)-\mathrm{SU}(3)$ transition are shown in Fig. 11 and for the $\mathrm{U}(5)-\mathrm{SO}(6)$ transition in Fig. 12. We see that the modifications induced by the presence of a fermion to the phase transition are more dramatic when the exchange interaction is added, especially in the location of the critical point $\xi_{i}^{c}$. The state 6 moves considerably to the right of $\xi_{B}^{c}$. In summary, QPTs in the presence of an odd fermion have a different behavior as a function of the control parameter $\xi$ 

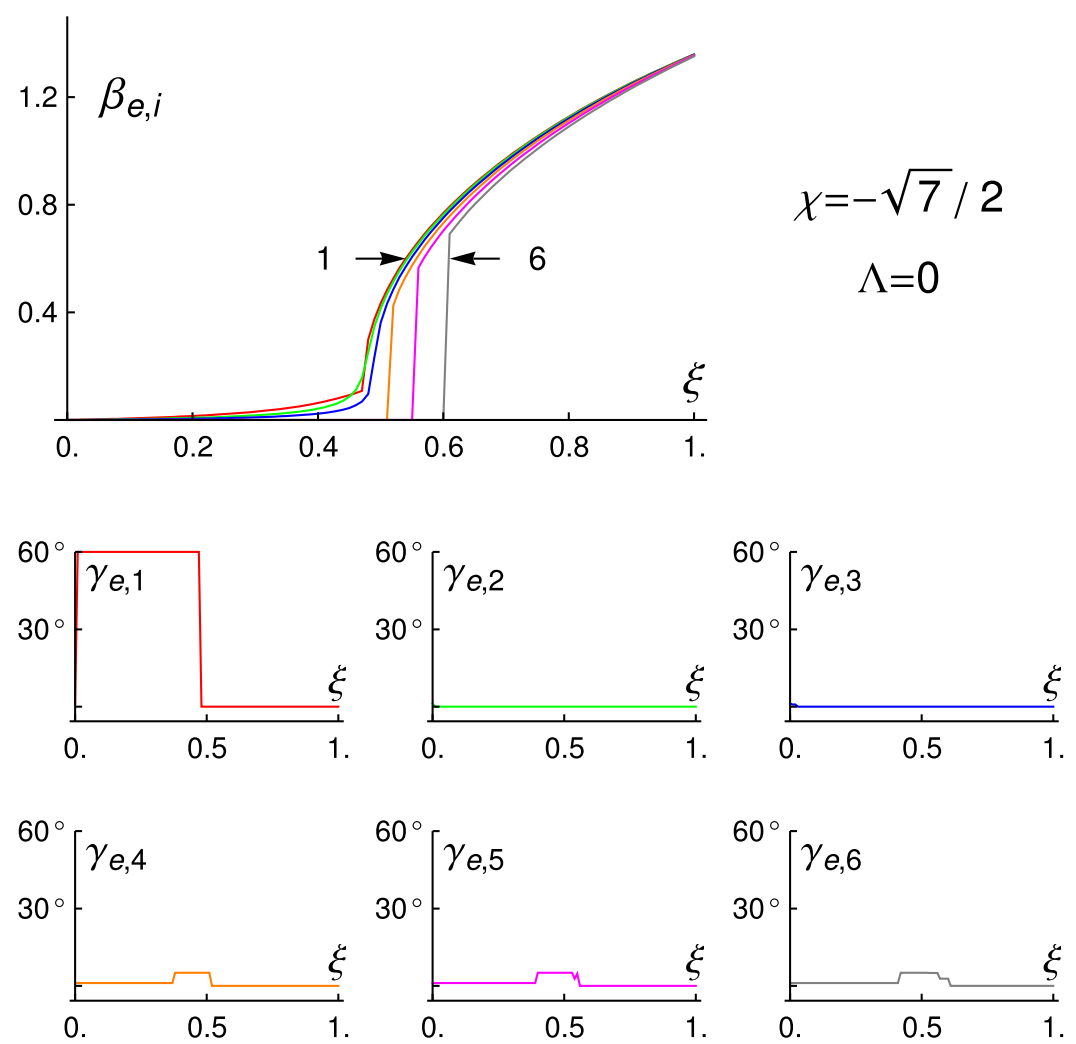

Fig. 9. Equilibrium values, $\beta_{e, i}$ (top part) and $\gamma_{e, i}$ (bottom part) as a function of the control parameter $\xi$ in the $\mathrm{U}(5)-\mathrm{SU}(3)$ transition $\left(\chi=-\frac{\sqrt{7}}{2}\right), \Lambda=0$ and $N=10$. States are labelled by the index $i=1, \ldots, 6$.
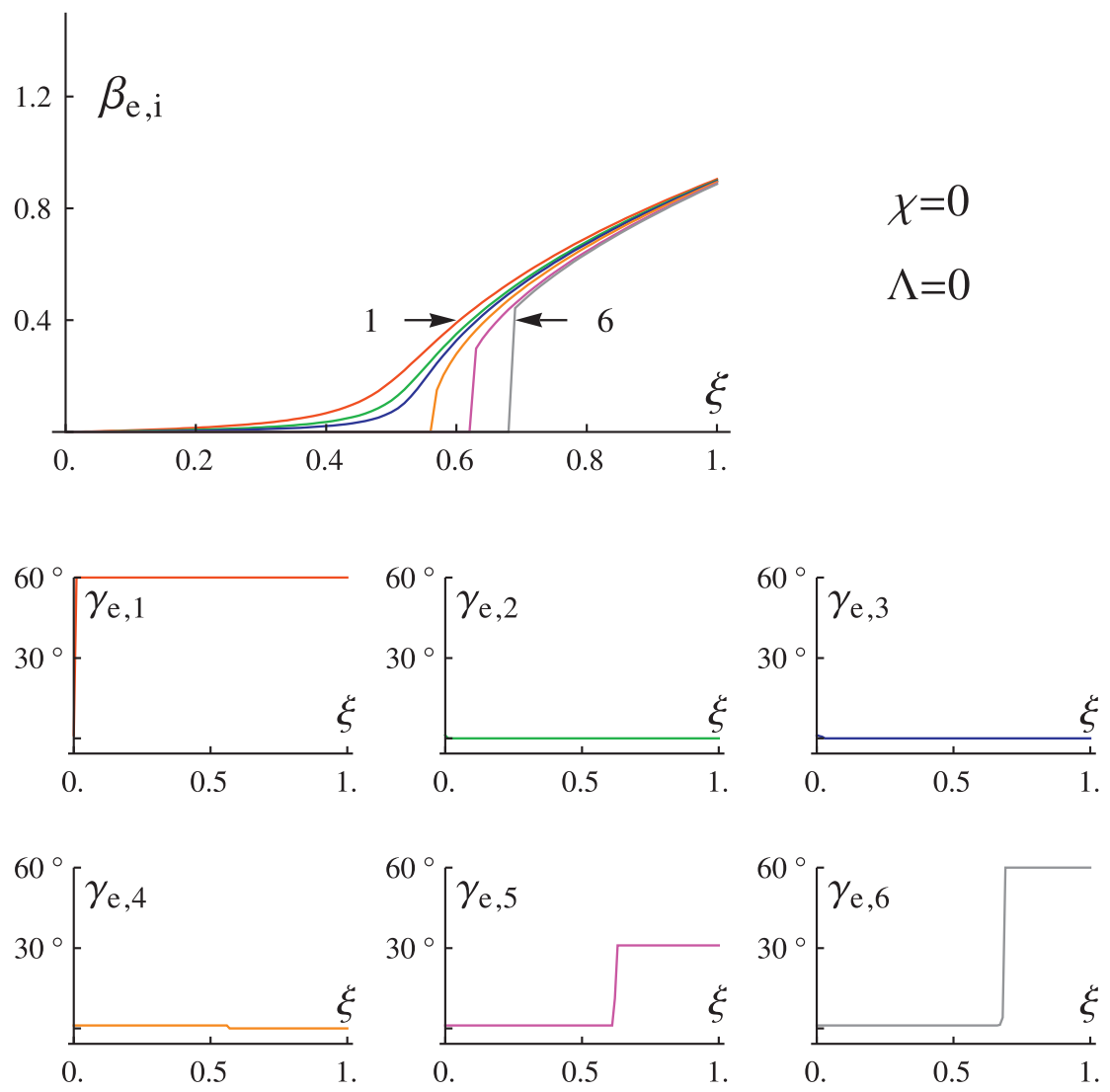

Fig. 10. Equilibrium values, $\beta_{e, i}$ (top part) and $\gamma_{e, i}$ (bottom part) as a function of the control parameter $\xi$ in the $\mathrm{U}(5)-\mathrm{SO}(6)$ transition $(\chi=0), \Lambda=0$ and $N=10$. Labelling as in Fig. 9. 

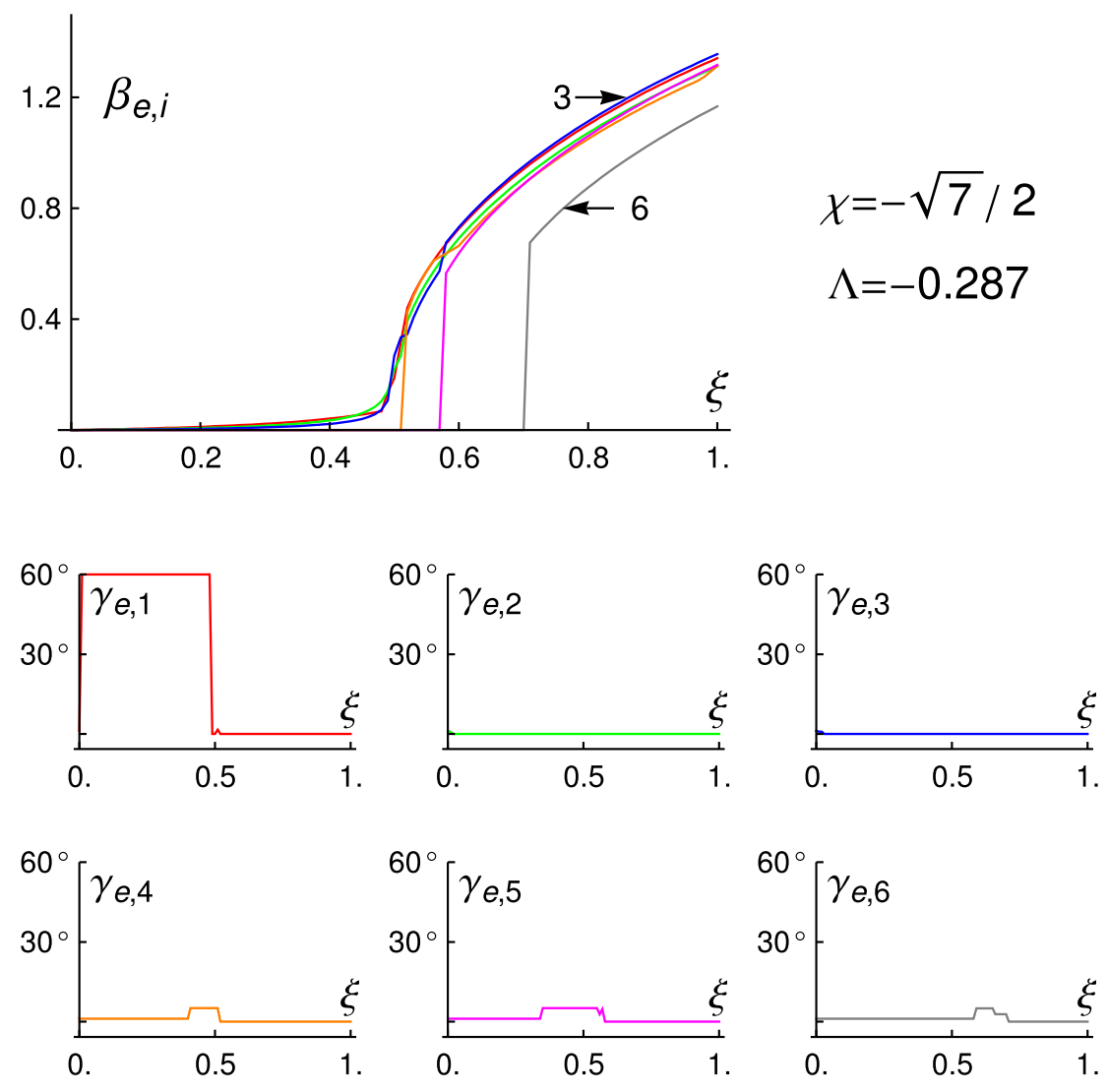

Fig. 11. Same as Fig. 9 but with $\Lambda=-0.287$.

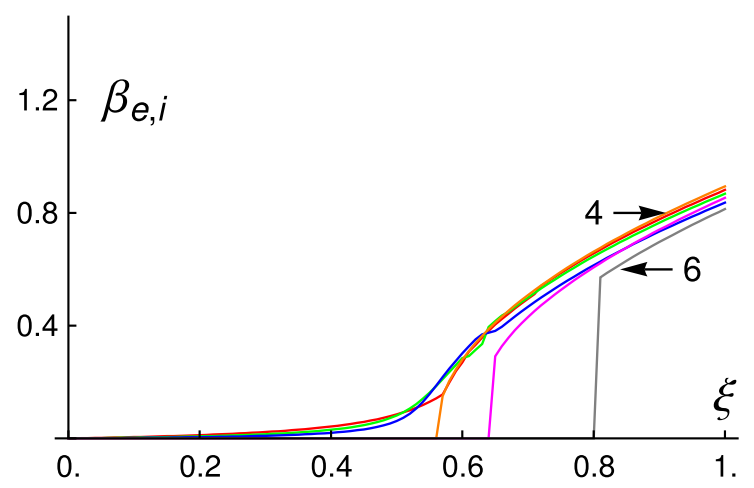

$$
\begin{gathered}
\chi=0 \\
\Lambda=-0.287
\end{gathered}
$$
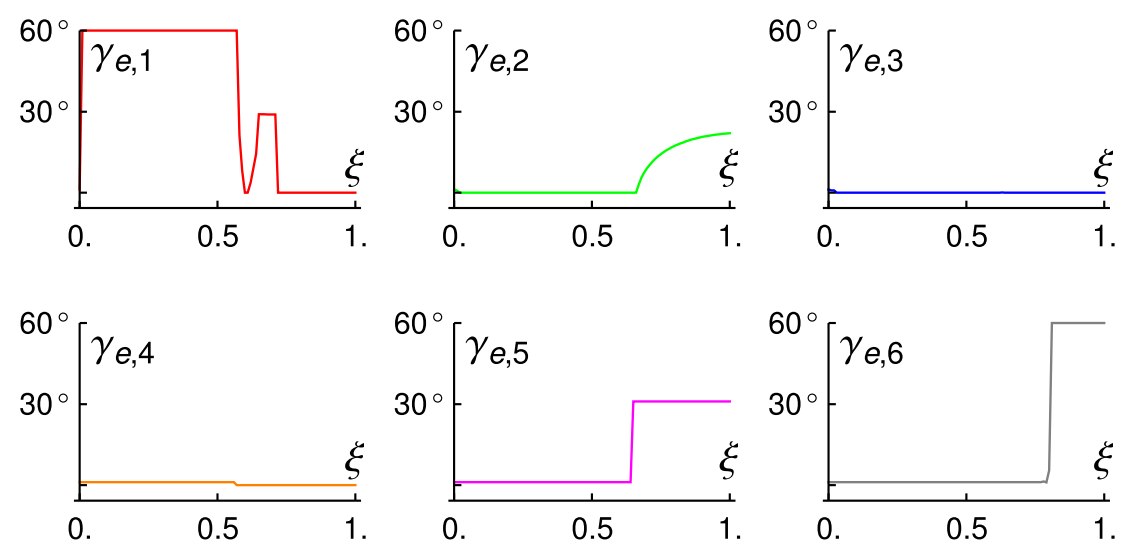

Fig. 12. Same as Fig. 10 but with $\Lambda=-0.287$. 
than in the purely bosonic system. The odd fermion acts as a catalyst for some states and as a retardative for others.

3.5. Single particle energies as a function of the control parameter $\xi$

Once the values of $\beta_{e, i}$ and $\gamma_{e, i}$ have been obtained, one can evaluate the single particle energies $e_{i}\left(N ; \beta_{e, i}, \gamma_{e, i}\right)$ as a function of $\xi$. Throughout this section we set the scale parameter $\varepsilon_{0}=1$ and $N=10$.

\subsubsection{Results for $\Lambda=0$}

Results for $\chi=-\frac{\sqrt{7}}{2}, \chi=0$ and $\Lambda=0$ are shown in Fig. 13. The discontinuities reflect the phase transition.

\subsubsection{Results for $\Lambda \neq 0$}

Results for $\chi=-\frac{\sqrt{7}}{2}, \chi=0$ and $\Lambda=-0.287$ are shown in Fig. 14. The single-particle energies are more spread in this case than when $\Lambda=0$ and have a different ordering.
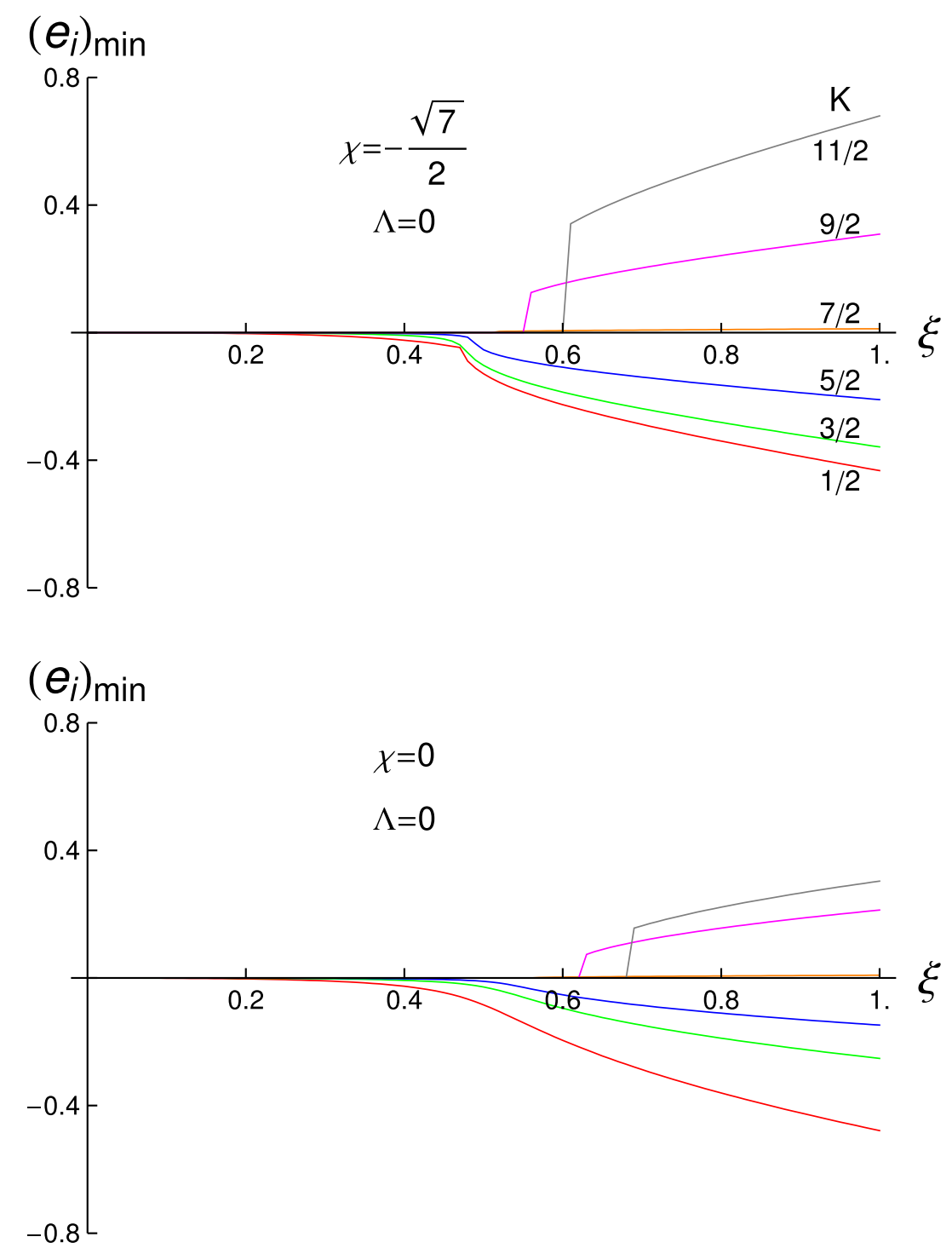

Fig. 13. Minimum single particle energies, $\left(e_{i}\right)_{\min }=-N \Gamma\left(\varepsilon_{i}\right)_{\min }$ as a function of the control parameter, $\xi$, in the $\mathrm{U}(5)-\mathrm{SU}(3)$ transition $\left(\chi=-\frac{\sqrt{7}}{2}\right)$ (top part) and in the $\mathrm{U}(5)-\mathrm{SO}(6)$ transition $(\chi=0)$ (bottom part), both with $\Lambda=0$. 
3.6. Total energies as a function of $\xi$

It is of interest to show also the results for the total energies $E_{i}\left(N ; \beta_{e, i}, \gamma_{e, i}\right)$ as a function of $\xi$. (The total energies depend also on $\chi$ and $\Lambda$ ). Throughout this section we set the scale parameter $\varepsilon_{0}=1$ and $N=10$.

\subsubsection{Results for $\Lambda=0$}

The effects of the odd particle in the total ground state energies are small, of order $1 / N$, as shown in Fig. 15.

\subsubsection{Results for $\Lambda \neq 0$}

The effects of the odd particle are also small here, as shown in Fig. 16 , for $\Lambda=-0.287$, although larger than in the case of $\Lambda=0$ since the reordering of the single particle states produces a lowering of some of them.
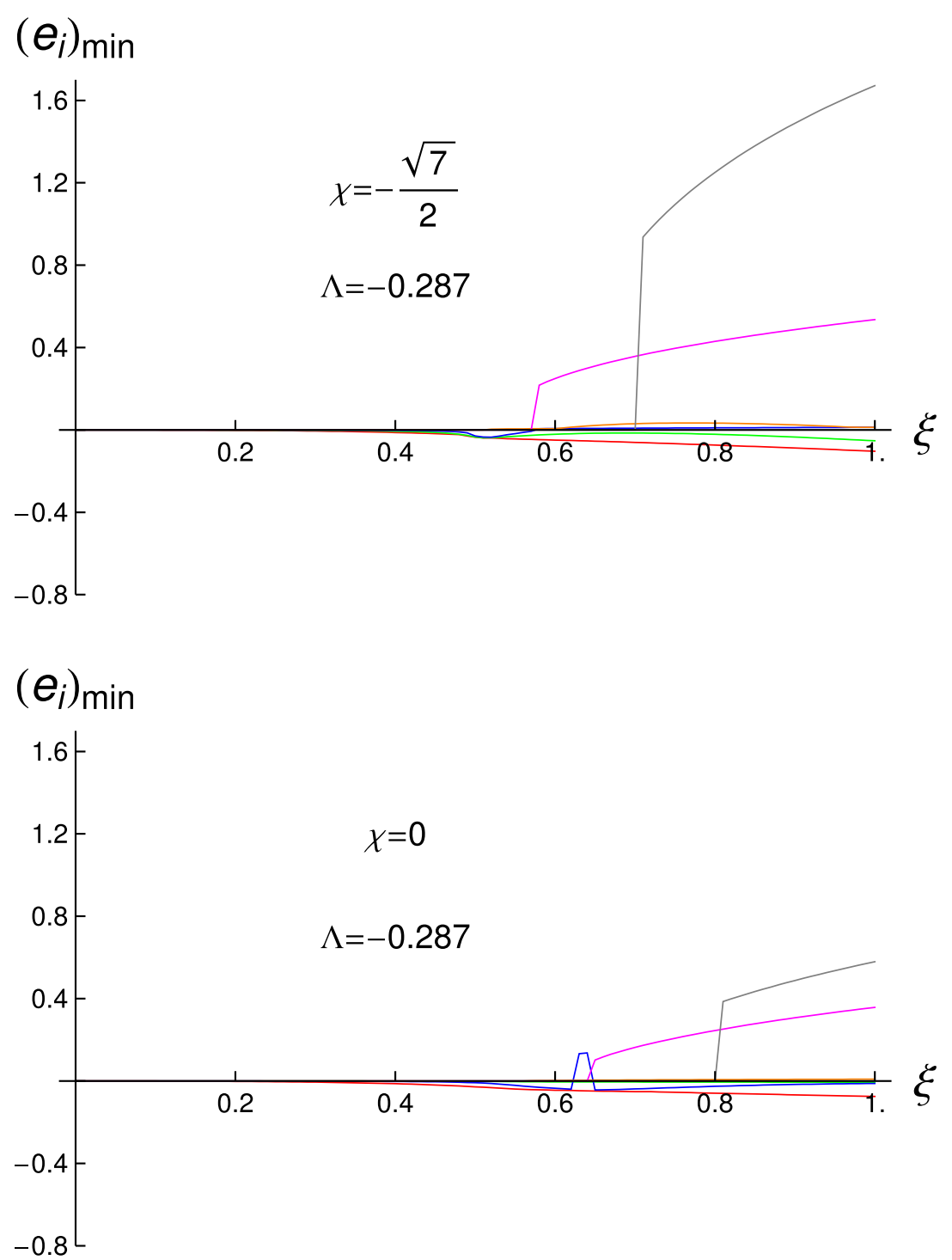

Fig. 14. Same as Fig. 13 but with $\Lambda=-0.287$. 


\section{Quantal analysis}

The crucial aspect in the study of quantum phase transitions is obviously the quantal analysis. This analysis must match the classical analysis of the previous section, valid in the limit $N \rightarrow \infty$. The quantal analysis for IBFM is done by diagonalizing numerically the Hamiltonian

$$
H=H_{B}+H_{F}+V_{B F}
$$

for finite $N$ using the program ODDA [42]. We take in this study $N=10$. Although this is a relatively small value, previous studies in the purely bosonic case have shown that the salient features of QPT are already apparent [16] at $N=10$.

The program ODDA uses a semi-microscopic version of IBFM in which (for a single fermion with angular momentum $j$ )

$$
\begin{aligned}
& H_{F}=\varepsilon_{j} \hat{n}_{j}, \\
& V_{B F}=V_{B F}^{M O N}+V_{B F}^{Q U A D}+V_{B F}^{E X C}
\end{aligned}
$$
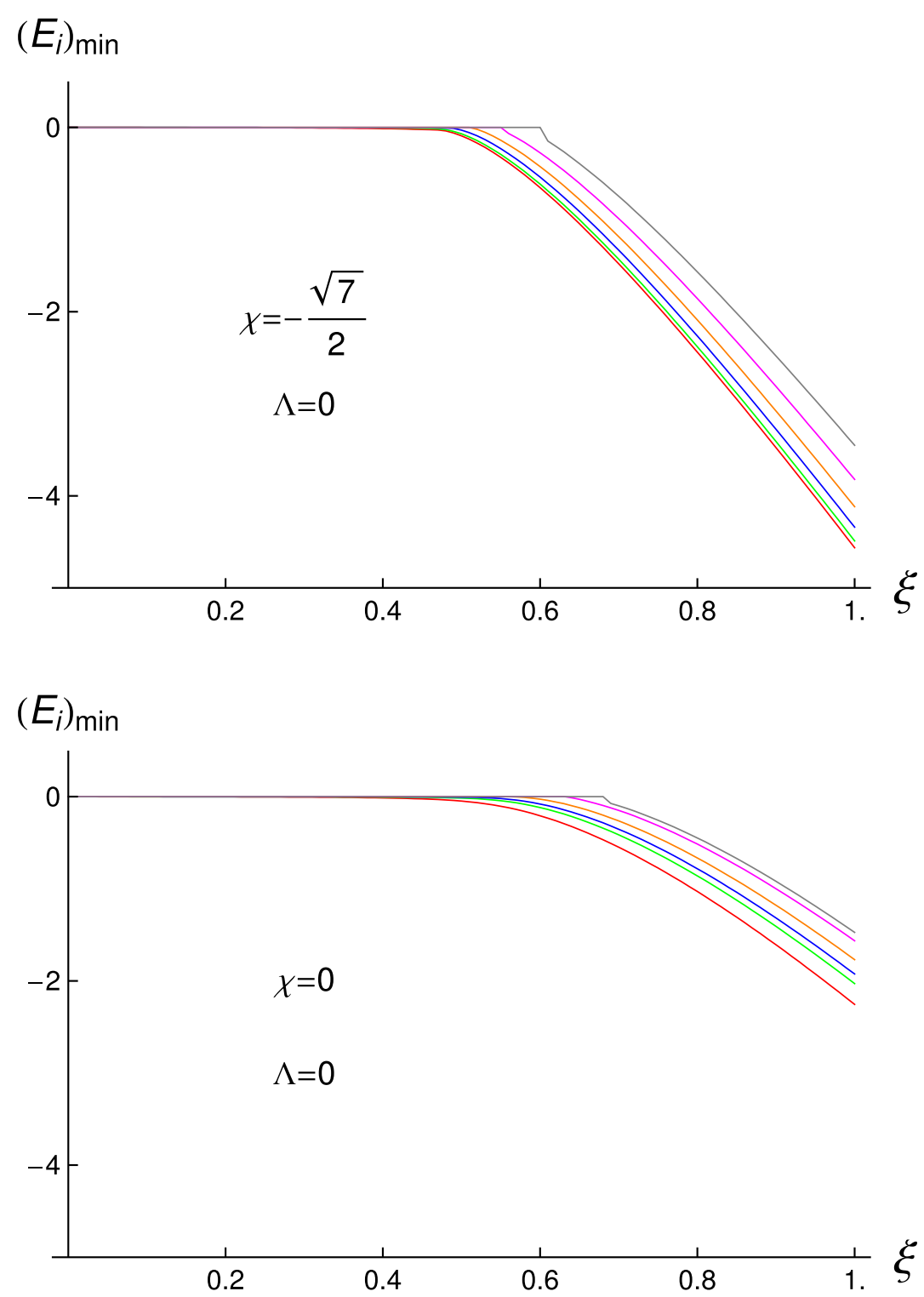

Fig. 15. Minimum total energies, $E_{i}(N ; \beta, \gamma ; \chi)$ as functions of the control parameter $\xi$, in the $\mathrm{U}(5)-\mathrm{SU}(3)$ transition $\left(\chi=-\frac{\sqrt{7}}{2}\right)$ (top part) and in the $\mathrm{U}(5)-\mathrm{SO}(6)$ transition $(\chi=0)$ (bottom part), both with $\Lambda=0$. 


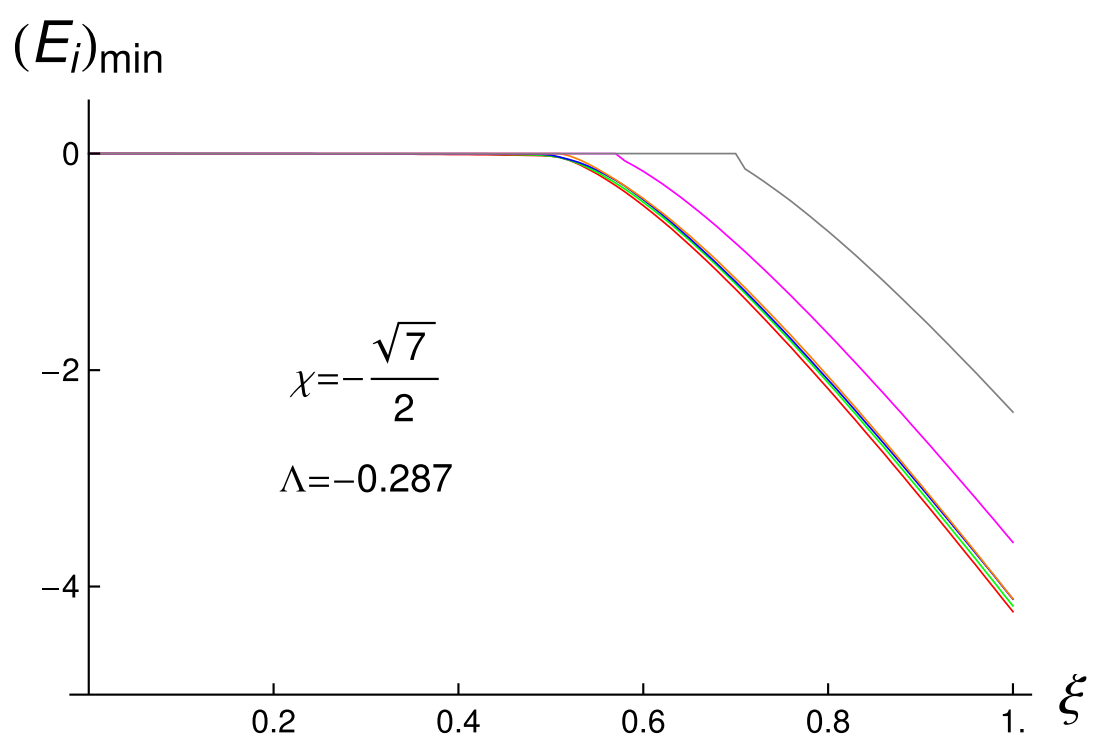

\section{$\left(E_{i}\right)_{\min }$}

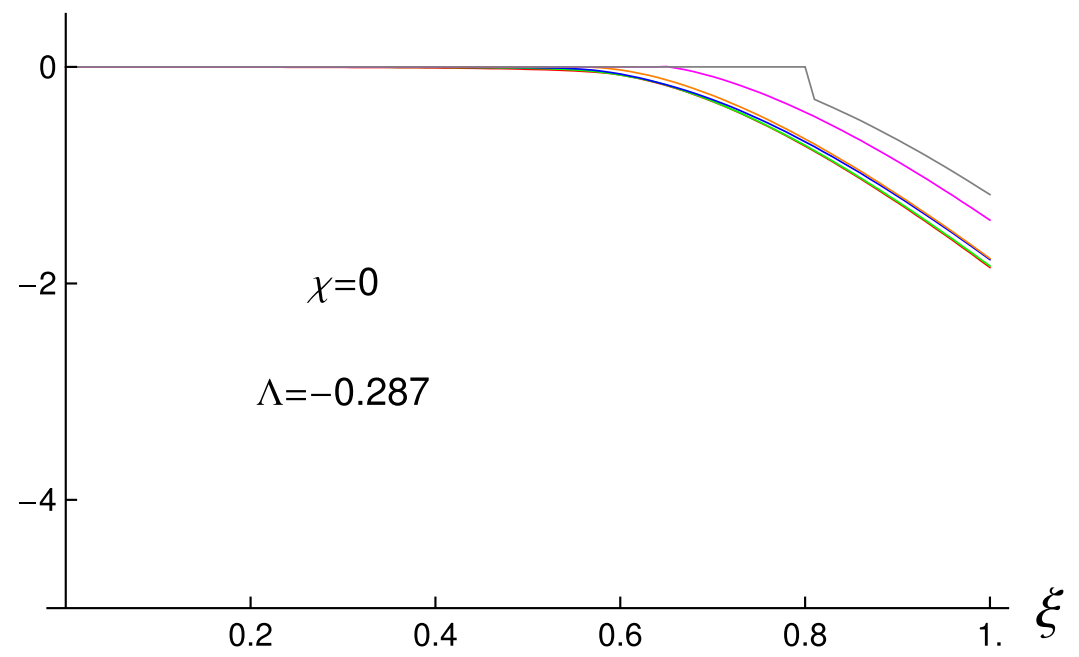

Fig. 16. Same as in Fig. 15 but for $\Lambda=-0.287$.

and

$$
\begin{aligned}
& V_{B F}^{M O N}=A_{s} \hat{n}_{d} \hat{n}_{j}, \\
& V_{B F}^{Q U A D}=\Gamma_{s}\left(u_{j}^{2}-v_{j}^{2}\right) Q_{j j}\left[\widehat{Q}^{\chi} \cdot \hat{q}_{j}+\hat{q}_{j} \cdot \widehat{Q}^{\chi}\right], \\
& V_{B F}^{E X C}=-\Lambda_{s} 8 \sqrt{5} u_{j}^{2} v_{j}^{2} Q_{j j}^{2} \frac{1}{\sqrt{2 j+1}}:\left[\left(d^{\dagger} \times \tilde{a}_{j}\right)^{(j)} \times\left(\tilde{d} \times a_{j}^{\dagger}\right)^{(j)}\right]_{0}^{(0)}:
\end{aligned}
$$

with $Q_{j j}$ defined in Eq. (30). In this semi-microscopic version, the Bose-Fermi interaction is given in terms of the BCS occupation probabilities, $u_{j}$ and $v_{j}$, with $u_{j}^{2}+v_{j}^{2}=1$. Comparing with the model Hamiltonian (3) of Section 2, we have the relationships

$$
\begin{aligned}
& A=A_{s}, \\
& \Gamma=\Gamma_{s} 2\left(u_{j}^{2}-v_{j}^{2}\right) Q_{j j}, \\
& \Lambda=-\Lambda_{s} 8 \sqrt{5} u_{j}^{2} v_{j}^{2} Q_{j j}^{2} /(2 j+1) .
\end{aligned}
$$




\subsection{The transition from spherical to axially deformed $(U(5)$ to $S U(3))$}

We study this transition by using a slightly modified form of the boson Hamiltonian

$$
\begin{aligned}
H_{B}^{U(5)-S U(3)} & =\varepsilon_{0}\left[(1-\xi) \hat{n}_{d}-\frac{\xi}{4 N}\left(\widehat{Q}^{\chi} \cdot \widehat{Q}^{\chi}+\frac{3}{8} \widehat{L} \cdot \widehat{L}\right)\right] \\
& =\varepsilon_{0}\left[(1-\xi) \widehat{C}_{1}(U(5))-\frac{\xi}{4 N} \frac{1}{2} \widehat{C}_{2}(S U(3))\right],
\end{aligned}
$$

with $\chi=-\frac{\sqrt{7}}{2}$. In (39) a term $\widehat{L} \cdot \widehat{L}$ has been added to the Hamiltonian of Eq. (2), where $\widehat{L}$ is the boson angular momentum operator. The combination $2\left[\widehat{Q} \cdot \widehat{Q}+\frac{3}{8} \widehat{L} \cdot \widehat{L}\right]$ is then the quadratic Casimir operator of SU(3). Also we denote by $\widehat{C}_{1}(g)$ and $\widehat{C}_{2}(g)$ Casimir operators of the first and the second order of the algebra $g$. The reason for using this boson Hamiltonian is that we want to isolate the intrinsic from the rotational part of the spectrum, in order to make the comparison between classical and quantal analysis straightforward. In the Bose-Fermi interaction, we set $A_{s}=0$ for the same reason as in the previous section.

\subsubsection{Correlation diagrams}

We first study the nature of the spectra in the presence of only a quadrupole interaction, $V_{B F}^{Q U A D}$, and set,

$$
\Gamma_{s}=\frac{\xi}{4 N} \varepsilon_{0}
$$

In the parameterization (37) we need to specify the values of the occupation probabilities, $v_{j}^{2}$. The correlation diagrams for $u_{j}^{2}=0, v_{j}^{2}=1$ (particle-like spectra) and $u_{j}^{2}=1, v_{j}^{2}=0$ (hole-like spectra) are shown in Fig. 17. Correlation diagrams describe how the energy levels evolve from one phase to the other as a function of the control parameter $\xi$. The phases are defined here by their symmetries, $\mathrm{U}(5)$ spherical, $\mathrm{SU}(3)$ axially deformed, and $\mathrm{SO}(6) \gamma$-unstable. Fig. 17 shows how rotational bands emerge from the spherical basis. It also displays in a clear fashion particle-hole conjugation, that is the transformation $(u \leftrightarrow v)$.

With the values of the BCS coefficients used in Fig. 17, the exchange interaction vanishes. To study the effect of the exchange interaction, we construct the correlation diagram as a function of $v_{j}^{2}$, Fig. 18 . This is the same as varying $\Lambda$, since $u_{j}^{2}=1-v_{j}^{2}$ and thus

$$
\Lambda=-\Lambda_{s} 8 \sqrt{5}\left(1-v_{j}^{2}\right) v_{j}^{2} Q_{j j}^{2} /(2 j+1) .
$$

As $v_{j}^{2}$ changes from 0 to 1 , we span the entire set of spectra from hole-like to particle-like. Particularly interesting in this figure are the spectra at half-filling, $v_{j}^{2}=0.5$. At this point, the quadrupole interaction vanishes, and the spectrum is given by the exchange interaction. It should be noted, however, that the diagram shown in Fig. 18 is an unconventional correlation diagram, since by varying $v_{j}^{2}$ we vary both the exchange and the quadrupole interaction, Eq. (37).

\subsubsection{Classical-quantal correspondence}

In addition to providing a description of the evolution of the quantal levels from one symmetry (phase) to another, correlation diagrams are also useful to test the classical-quantal correspondence. In Section 3, the single-particle energies for a fermion with angular momentum $j$ in the presence of the boson condensate of Eq. (6) were calculated classically. For $\gamma=0^{\circ}$ they were given by Eqs. (24) and (25), as a function of $\beta$ and for any $\chi$. When the bosons have $\mathrm{SU}(3)$ symmetry, $\chi=-\frac{\sqrt{7}}{2}$, and in the limit $N \rightarrow \infty$, the equilibrium deformation is $\beta_{e}=\sqrt{2}$. Inserting these values in Eq. (24) we obtain the classical result

$$
\begin{aligned}
\lambda_{K}^{S U(3)}= & -N \Gamma \sqrt{2} \sqrt{5} P_{j}\left[3 K^{2}-j(j+1)\right] \\
& -N \Lambda \frac{2}{3}(2 j+1) P_{j}^{2}\left[3 K^{2}-j(j+1)\right]^{2} .
\end{aligned}
$$



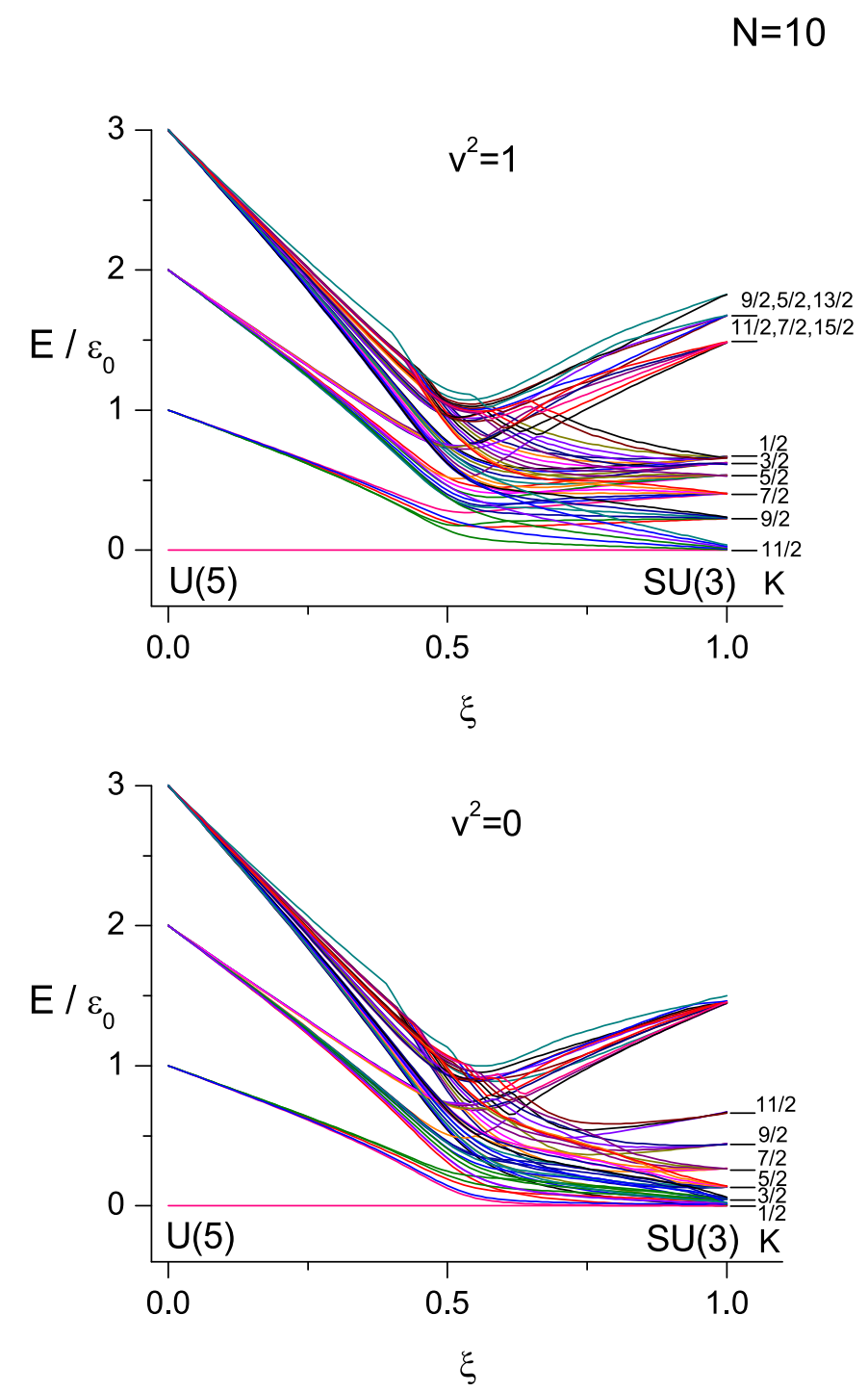

Fig. 17. Correlation diagram for a $j=11 / 2$ particle coupled to a system of $(s, d)$ bosons undergoing a $\mathrm{U}(5)-\mathrm{SU}(3)$ phase transition. Top part $v^{2}=1$, bottom part $v^{2}=0$. The interaction is purely quadrupole.

An analytic quantal calculation of the single-particle energies for bosons with SU(3) symmetry in the limit of large $N$ can also be done. The bosonic ground state wave function, with good SU(3) quantum numbers, can be written as $\left|[N],(2 N, 0), K_{c}=0, L, M\right\rangle$ [7]. States for a fermion with angular momentum $j$ coupled to the bosonic ground state can be written as $\left|[N],(2 N, 0), K_{c}=0 ; j, K_{j} ; K=K_{j}, J, M\right\rangle$. A long but straightforward calculation of the matrix elements of the interaction $V_{B F}=V_{B F}^{Q U A D}+V_{B F}^{E X C}$, with $V_{B F}^{Q U A D}$ and $V_{B F}^{E X C}$ as in Eq. (37), gives [43]

$$
\lim _{N \rightarrow \infty}\left\langle[N],(2 N, 0), 0 ; j, K_{j} ; K, J, M\left|V_{B F}\right|[N],(2 N, 0), 0 ; j, K_{j} ; K, J, M\right\rangle=\lambda_{K}^{S U(3)} .
$$

Here the parameters $\Gamma$ and $\Lambda$ in the expression for $\lambda_{K}^{S U(3)}$, Eq. (42), are related to the parameters of $V_{B F}$, Eq. (37), by means of Eqs. (38) and (40). Specifically, $\Gamma=2 \Gamma_{s}\left(1-2 v_{j}^{2}\right) Q_{j j}, \Gamma_{s}=\frac{\xi}{4 N} \varepsilon_{0}$ and $\Lambda$ is given in Eq. (41). It should also be noted that in the limit $N \rightarrow \infty$, the expectation value of $V_{B F}$ is independent of the the total angular momentum $J$.

Both the explicit formula Eq. (43) and the independence on $J$ can be checked numerically. In Fig. 17 top, obtained with $\Gamma=-\frac{\xi}{4 N}\left(2 \varepsilon_{0}\right) Q_{j j}$ and $\Lambda=0$, one can see that at $\xi=1, \mathrm{SU}(3)$ symmetry, all rotational levels built on the intrinsic state $K$, converge to a single value, as seen on the right-hand side of the figure, and that the energies of the intrinsic states can be described to a good approximation by Eq. 


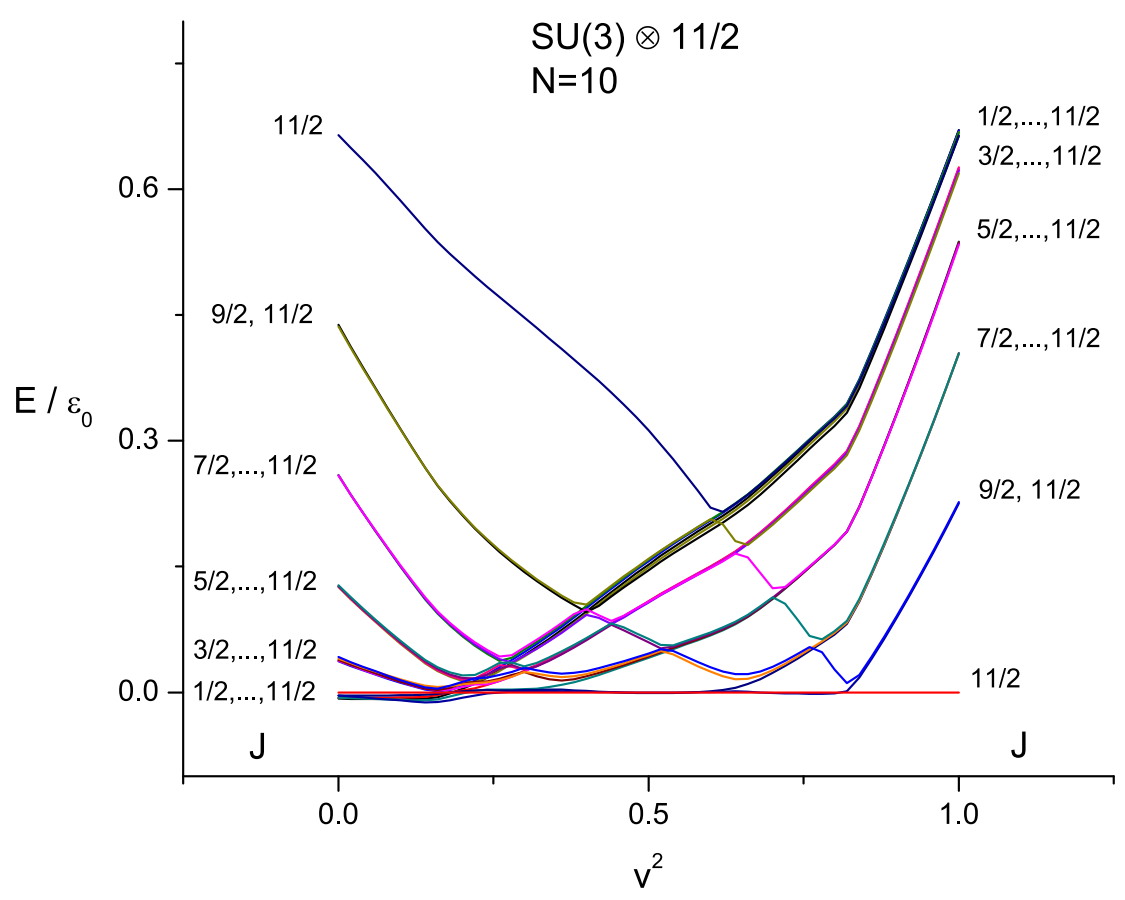

Fig. 18. Correlation diagram for a $j=11 / 2$ particle coupled to a system of $(s, d)$ bosons with quadrupole, $V_{B F}^{\text {QUAD }}, \chi=-\frac{\sqrt{7}}{2}$, and exchange, $V_{B F}^{E X C}$, interaction parametrized as in Eq. (37), with $\Lambda_{s} / \Gamma_{s}=3$, as a function of the filling probability $v^{2} \equiv v_{j}^{2}$ of the single particle state. States are labelled by the total angular momentum $J$.

(42). Since the figure represents the results for $N=10$, one can see that small deviations occur, especially for the band with $K=1 / 2$, but these deviations are relatively small and the large $N$ limit appears to be already reached. The same conclusion applies to Fig. 17 bottom obtained with $\Gamma=+\frac{\xi}{4 N}\left(2 \varepsilon_{0}\right) Q_{j j}$, and $\Lambda=0$. Flipping the sign of $\Gamma$ (particle-hole conjugation) reverses the ordering of the $K$ states but the energies are still given by Eq. (42). (The bottom part of Fig. 17 is also consistent with the top part of Fig. 3, once the relationship $\Gamma=2 \Gamma_{s} Q_{j j}$ is taken into account.)

The correspondence between classical and quantal calculation is exact at $\xi=1, \mathrm{SU}(3)$ symmetry, and $N \rightarrow \infty$. As one moves away from $\xi=1$, or for finite $N$, it becomes approximate. Nonetheless, rotational bands can still be identified for $\xi_{c} \leqslant \xi \leqslant 1$. Below the critical value, the characterization of states by a $K$ quantum number is no longer possible. For this region, $0 \leqslant \xi \leqslant \xi_{c}$, a straightforward comparison between classical and quantal calculation is no longer possible and one must resort to a numerical calculation both classical, Section 3, and quantum-mechanical, Section 4.

\subsubsection{Ground state energy}

The ground state energy is a key indicator of phase transitions. The ground state energy, $E_{0}$, its first, $\frac{\partial E_{0}}{\partial \xi}$, and second, $\frac{\partial^{2} E_{0}}{\partial \xi^{2}}$, derivatives with respect to $\xi$ are shown in Fig. 19 (particle-like, $v_{j}^{2}=1$ ). (The energy $E_{0}$ in this figure is in units of the scale factor $\varepsilon_{0}$, taken to be $\varepsilon_{0}=1$ and $N=10$ ). For hole-like spectra, $v_{j}^{2}=0$, we have a similar behavior, as well as in the case in which $\Lambda \neq 0$. The quantal result for $E_{0}$ should be compared with the classical result, $\left(E_{0}\right)_{\min }$, shown in the top part of Fig. 15 , where $\left(E_{0}\right)_{\min }$ is the energy of the lowest state at the minimum. Within $1 / N$ corrections, the two results agree (classical-quantal correspondence). Fig. 19 is similar to Fig. 7.3 of [21] for the purely bosonic case. Although the value of $N$ used here is too small to distinguish between first and second order transition, with discontinuities in $\frac{\partial E_{0}}{\partial \xi}$ and $\frac{\partial^{2} E_{0}}{\partial \xi^{2}}$, respectively, nonetheless precursors of the QPT are clearly seen at $\xi=\xi_{c} \cong 0.5$.

\subsubsection{Quantal order parameters}

The equilibrium deformations $\beta_{e, i}, \gamma_{e, i}$ are the classical order parameters. As quantal order parameters we consider here only the expectation value of $\hat{n}_{d}$ in the states $i=1, \ldots, 6$ (first quantal order parameter), $v_{i}^{(1)}$ 

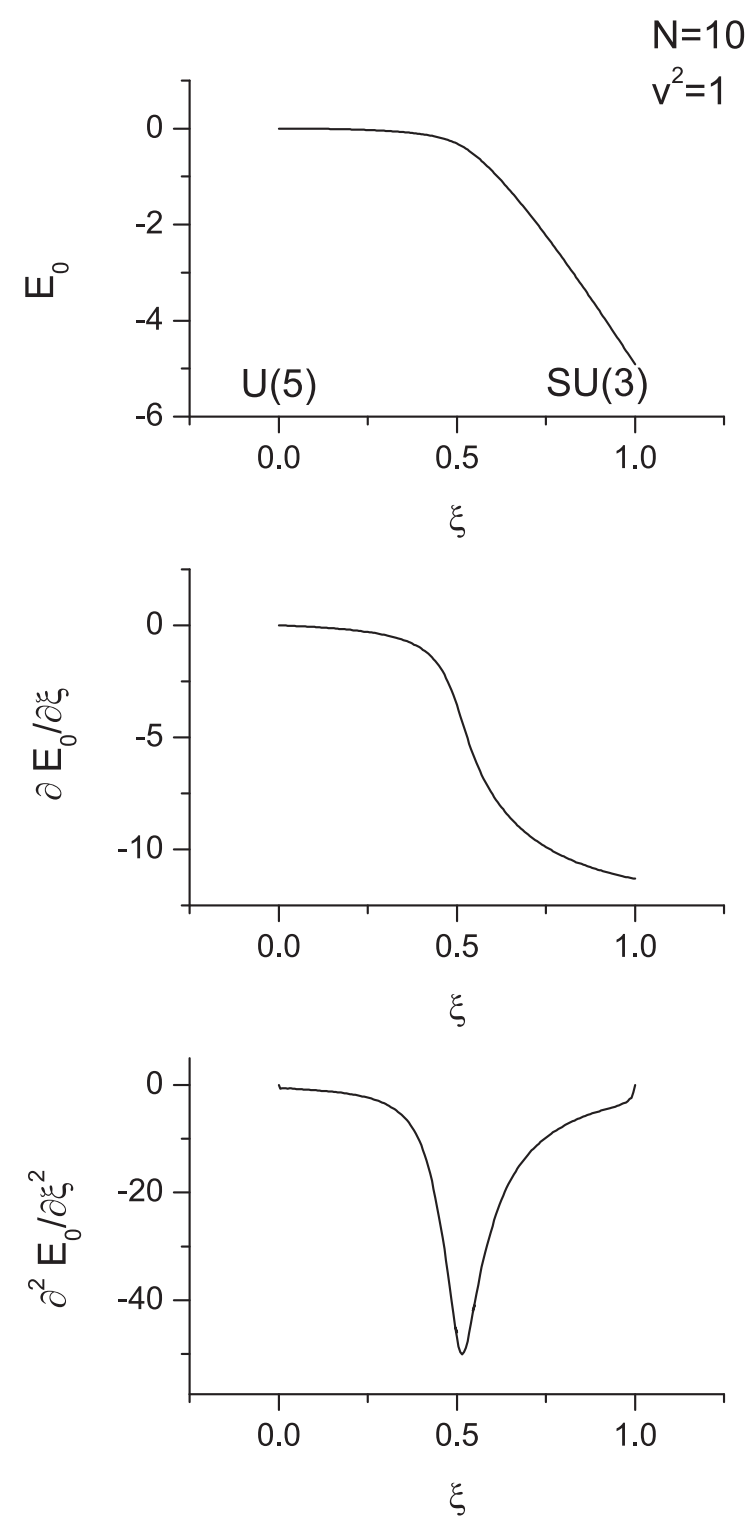

Fig. 19. The ground state energy, $E_{0}$, top, its first derivative, $\frac{\partial E_{0}}{\partial \xi}$, center, and its second derivative, $\frac{\partial^{2} E_{0}}{\partial \xi^{2}}$, bottom, for a $j=11 / 2$ particle coupled to a system of $(s, d)$ bosons undergoing a $\mathrm{U}(5)-\mathrm{SU}(3)$ transition.

$$
v_{i}^{(1)}=\frac{\left\langle\psi_{i}\left|\hat{n}_{d}\right| \psi_{i}\right\rangle}{N}
$$

This is shown in Fig. 20 top part. Since the order parameters $v_{i}^{(1)}$ are related to the square of the classical order parameters $\beta_{e, i}$, this figure is related to Fig. 9 to which it corresponds in the limit $N \rightarrow \infty$. The derivative of $v_{1}^{(1)}$ in the ground state, $\frac{\partial v_{i}^{(1)}}{\partial \xi^{\prime}}$ is also shown in Fig. 20 bottom part. (This quantity diverges when $N \rightarrow \infty$.) From this figure one sees clearly that the transition is made sharper by the presence of the fermion for some states, $11 / 2,9 / 2,7 / 2$, while is made smoother for others, $5 / 2,3 / 2,1 / 2$, a result already seen in the classical analysis.

4.2. The transition from spherical to $\gamma$-unstable $(U(5)-S O(6))$

We study this transition by using the standard form of the transitional boson Hamiltonian

$$
H_{B}^{\mathrm{U}(5)-\mathrm{SO}(6)}=\varepsilon_{0}\left[(1-\xi) \hat{n}_{d}-\frac{\xi}{4 N} \widehat{Q}^{\chi=0} \cdot \widehat{Q}^{\chi=0}\right],
$$



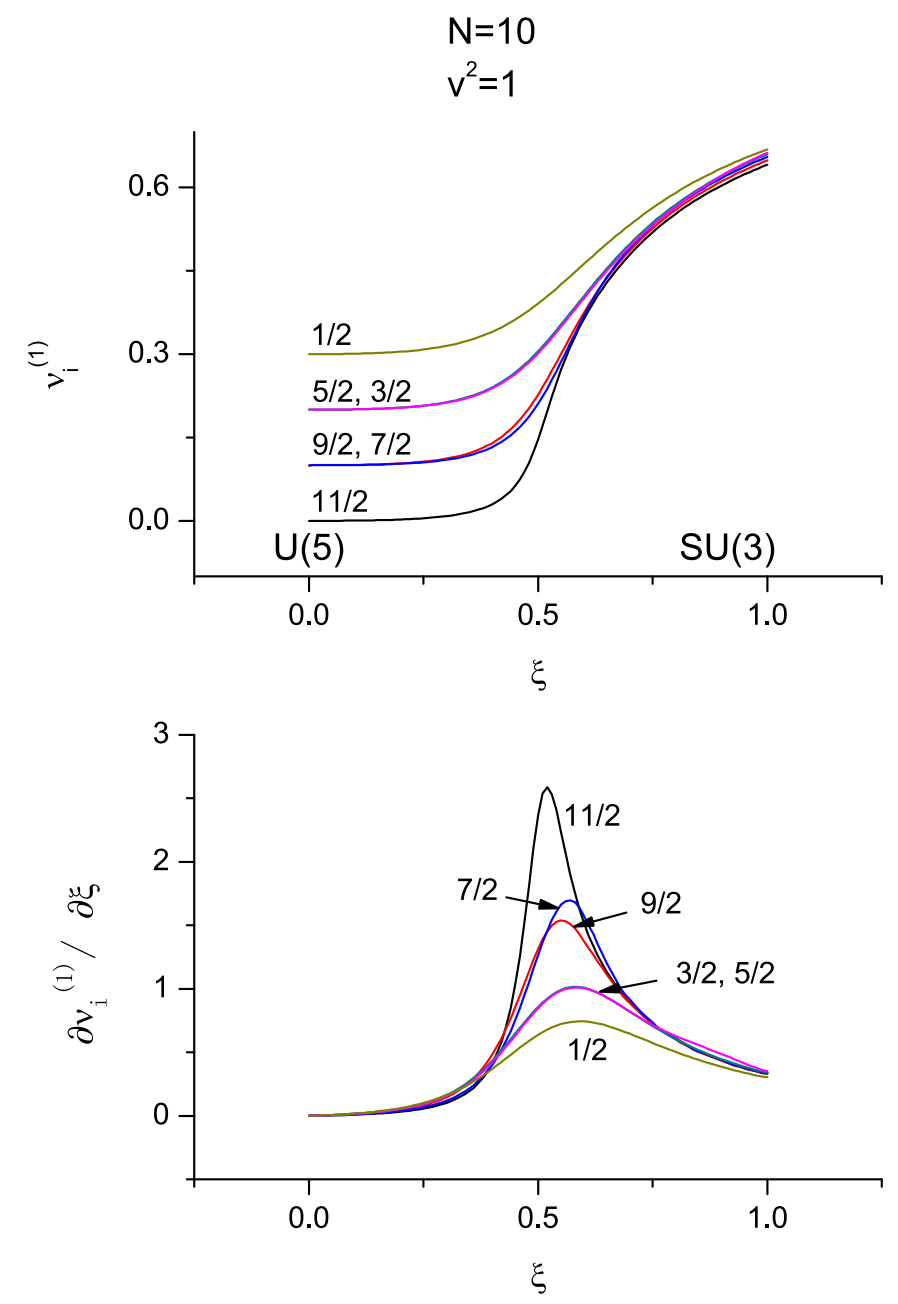

Fig. 20. The quantal order parameters, $v_{i}^{(1)}$, Eq. (44), as a function of the control parameter, $\xi$ (top part). The expectation values of the number of $d$ bosons, $\left\langle\hat{n}_{d}\right\rangle$, for the lowest states of a given total angular momentum, $J=11 / 2, \ldots, 1 / 2$ for a $j=11 / 2$ particle coupled to a system of $(s, d)$ bosons undergoing a $\mathrm{U}(5)-\mathrm{SU}(3)$ transition. Particle-like spectra, $v^{2}=1$, and no exchange interaction. The derivative $\frac{\partial v_{i}^{(1)}}{\partial \xi}$ as a function of $\xi$ (bottom part).

with $\chi=0$. We set $A_{s}=0$ as in the previous section and $\Gamma_{s}=\frac{\xi}{4 N} \varepsilon_{0}$ as in Eq. (40).

\subsubsection{Correlation diagram}

The correlation diagram for this phase transition is shown in Fig. 21. In this case, there is no clear separation between intrinsic and rotational motion, since $\widehat{Q}^{\chi=0} \cdot \widehat{Q}^{\chi=0}$ is not a Casimir operator of $\mathrm{SO}(6)$, and thus the states at $\xi=1$ do not coalesce into a single point as in Fig. 17. (We have also done a study in which $\widehat{C}_{2}(\mathrm{SO}(6))$ is used instead of $\widehat{Q}^{\chi=0} \cdot \widehat{Q}^{\chi=0}$. These two operators are related by $\widehat{C}_{2}(\mathrm{SO}(6))=\widehat{Q}^{\chi=0} \cdot \widehat{Q}^{\chi=0}+\widehat{C}_{2}(\mathrm{SO}(5))$. However, using the Casimir operator, makes the figure at the end point $\xi=1$ very crowded, since all states arising from a given $S O(6)$ representation collapse to zero energy, and, for this reason, we prefer to plot in Fig. 21 the results with the Hamiltonian (45).

The effect of the exchange interaction is studied by constructing the correlation diagram as a function of $v_{j}^{2}$, Fig. 22. A remarkable property of this diagram is the symmetry under particle-hole conjugation. The diagram is symmetric around half-filling. This is unlike the case of the $\mathrm{U}(5)-\mathrm{SU}(3)$ transition.

\subsubsection{Ground state energy}

The ground state energy, $E_{0}$, its first and second derivative are shown in Fig. 23, U(5)-SO(6) transition. This figure is similar to Fig. 19, U(5)-SU(3) transition, and as mentioned in Section 4.1.3, it is not possible to distinguish whether the transition is first or second order. In order to do so, one must go to much larger values of $N$, as done in the purely bosonic case [22]. However, the fact that the quantity 


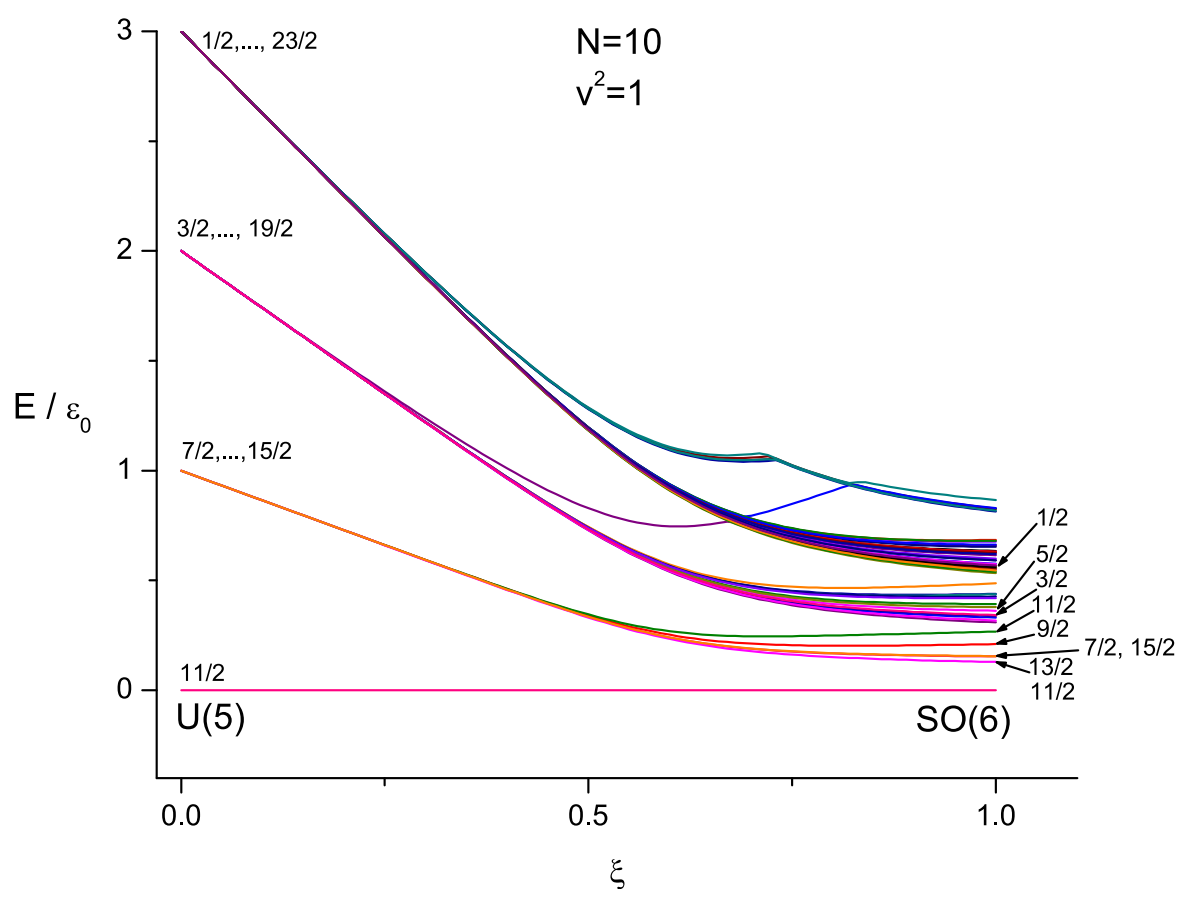

Fig. 21. Correlation diagram for a $j=11 / 2$ particle coupled to a system of $(s, d)$ bosons undergoing an $\mathrm{U}(5)-\mathrm{SO}(6)$ transition. The interaction is purely quadrupole. There is no difference in this case between particle-like $\left(v^{2}=1\right)$ and hole-like $\left(v^{2}=0\right)$ spectra.

$\frac{\partial^{2} E_{0}}{\partial \xi^{2}}$ is smoother in Fig. 23 than in Fig. 19 supports the conclusion that the ground state transition is first order for $\mathrm{U}(5)-\mathrm{SU}(3)$ and second order for $\mathrm{U}(5)-\mathrm{SO}(6)$, since the second derivative of $E_{0}$ diverges (or not) for first (or second) order transitions in the limit $N \rightarrow \infty$.

\section{Experimental evidence}

Even-even nuclei in the mass region $A \sim 150$ are known to experience a first order quantum phase transition $(\mathrm{U}(5)-\mathrm{SU}(3))$ at neutron number 90 . The evidence for this statement comes from the anal-

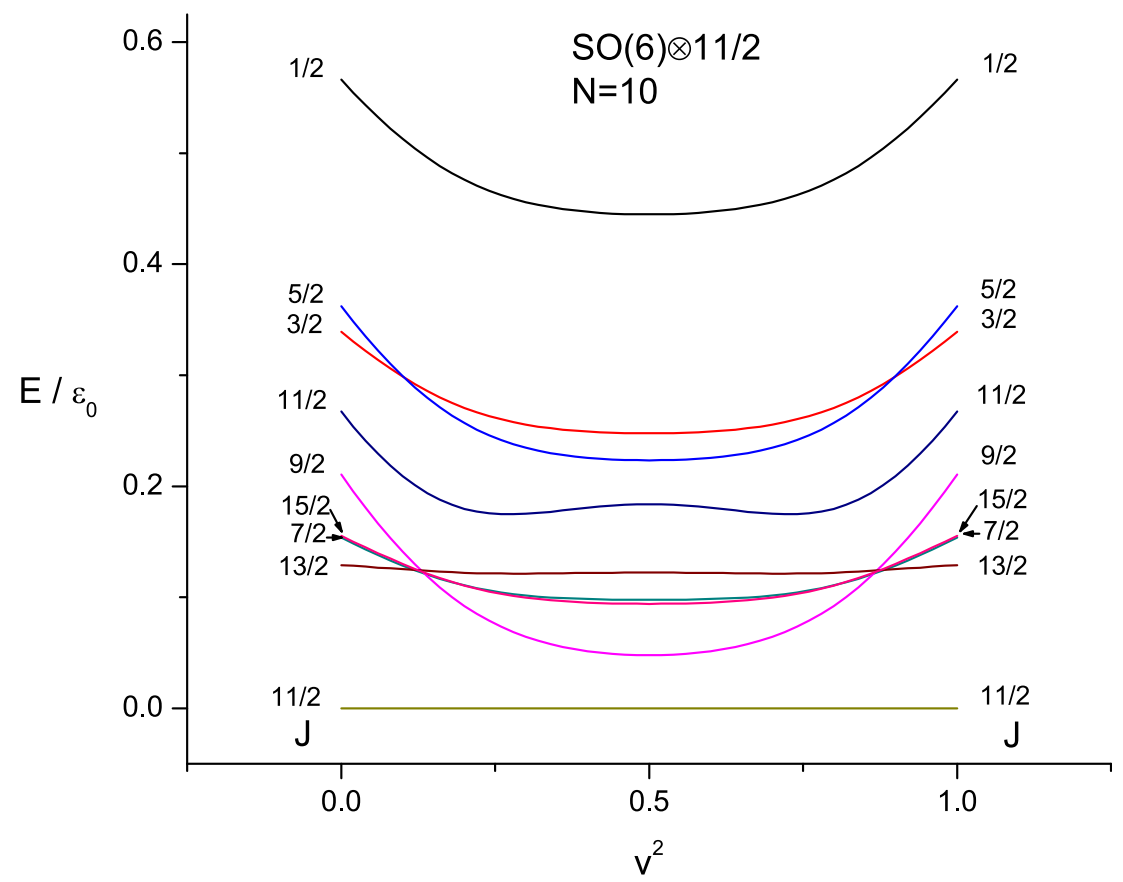

Fig. 22. Same as Fig. 18 but with $\chi=0$. 

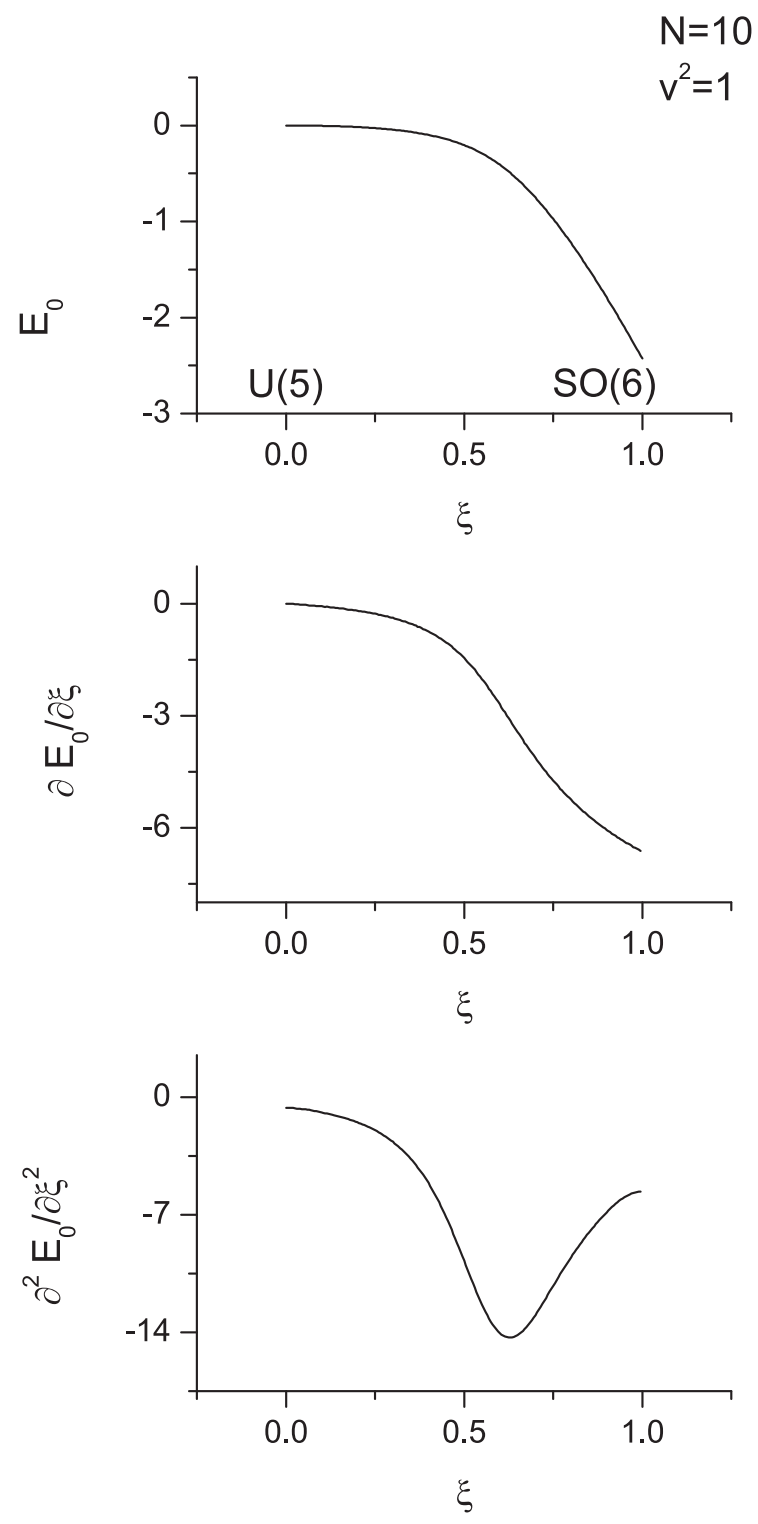

Fig. 23. Same as Fig. 19 but for the $\mathrm{U}(5)-\mathrm{SO}(6)$ transition.

ysis of (i) the excitation spectrum which displays a gap, $\Delta=E\left(0_{2}^{+}\right)-E\left(0_{1}^{+}\right)$; (ii) the two neutron separation energies, $S_{2 n}(N)=-\left[E_{0}(N+1)-E_{0}(N)\right]$, proportional to the derivative of the ground state energy, $E_{0}$, with respect to the control parameter, $\xi$, i.e. $\frac{\partial E_{0}}{\partial \xi}$, and (iii) the $B(E 2 ; 0 \rightarrow 2)$ values, proportional to the square of the order parameter $\left\langle\hat{n}_{d}\right\rangle^{2}[14,25,34]$. In odd-even nuclei, the first two quantities can be easily measured, while the last quantity, $B\left(E 2 ; J_{g . s .} \rightarrow J^{\prime}\right)$ is more difficult to measure due to the fragmentation of the $B(E 2)$ strength from the ground state to several states $J^{\prime}$. In this paper, we therefore analyze the first two quantities and show that both display the features expected for a QPT, as described in Sections 3 and 4.

\subsection{Excitation spectrum}

Odd-proton nuclei in the region of $A \sim 150$ offer a unique opportunity to study QPT in Bose-Fermi systems, because of the occurrence of the unique parity state $h_{11 / 2}$ near the Fermi surface. We therefore analyze the negative parity states of the odd-proton nuclei, ${ }_{61} \mathrm{Pm}_{86-92},{ }_{63} \mathrm{Eu}_{86-92}$, and ${ }_{65} \mathrm{~Tb}_{86-92}$. A realistic calculation for these nuclei can be done within the framework of IBFM, with an Hamiltonian $H=H_{B}+H_{F}+V_{B F}$. The IBM Hamiltonian $H_{B}$ is written as [7] 


$$
\begin{aligned}
H_{B}= & \varepsilon \hat{n}_{d}+\sum_{L=0,2,4} c_{L} \frac{1}{2} \sqrt{2 L+1}\left[\left(d^{\dagger} \times d^{\dagger}\right)^{(L)} \times(\tilde{d} \times \tilde{d})^{(L)}\right]_{0}^{(0)} \\
& +\frac{1}{\sqrt{2}} v_{2}\left[\left(d^{\dagger} \times d^{\dagger}\right)^{(2)} \times(\tilde{d} \times s)^{(2)}+\left(s^{\dagger} \times d^{\dagger}\right)^{(2)} \times(\tilde{d} \times \tilde{d})^{(2)}\right]_{0}^{(0)} \\
& +\frac{1}{2} v_{0}\left[\left(d^{\dagger} \times d^{\dagger}\right)^{(0)} \times(s \times s)^{(0)}+\left(s^{\dagger} \times s^{\dagger}\right)^{(0)} \times(\tilde{d} \times \tilde{d})^{(0)}\right]_{0}^{(0)} .
\end{aligned}
$$

In order to perform a calculation of the odd-proton nuclei indicated above, we need the Hamiltonian parameters of the even-even nuclei ${ }_{60} \mathrm{Nd}_{86-92},{ }_{62} \mathrm{Sm}_{86-92,}{ }_{64} \mathrm{Gd}_{86-92}$. We take these parameters from previous studies. In these studies, first a calculation in the Proton-Neutron Interacting Boson Model (IBM-2) is done with parameters given in Table 1 . The input parameters in IBM, $\varepsilon, c_{L}(L=0,2,4), v_{2}$, $v_{0}$, Eq. (46) are then calculated from these by a projection technique [43].

In the case of the unique parity configuration $h_{11 / 2}$ there is only one single particle energy, $\varepsilon_{j}$, which we take as $\varepsilon_{j}=0$. The Bose-Fermi interaction (37) is specified by the parameters $A_{s}, \Gamma_{s}$ and $\Lambda_{s}$ and $v_{j}^{2}$, three of which are independent. Using the semi-microscopic theory, one can extract the occupation probabilities $v_{j}^{2}$. The parameters used in the present analysis are given in Table 2. For ${ }_{63} \mathrm{Eu}$ and ${ }_{61} \mathrm{Pm}$ isotopes, they are the same parameters used by Scholten and Blasi [29] and Scholten and Ozzello [44], respectively, in previous studies of these isotopes. For the ${ }_{65} \mathrm{~Tb}$ isotopes we scale the latter parameters. Our calculated spectra are shown in Figs. 24-26, where they are compared with the available experimental data [45]. One can see very clearly the phase transition occurring between neutron numbers 88 and 90 both in the theoretical (left) and in the experimental (right) spectra.

Table 1

Parameters of IBM-2 used in the calculation of the even-even nuclei ${ }^{146-150} \mathrm{Nd},{ }^{148-152} \mathrm{Sm},{ }^{150-156} \mathrm{Gd}$.

\begin{tabular}{llllllrrr}
\hline & $N_{\pi}$ & $N_{v}$ & $\varepsilon$ & $\kappa$ & $\chi_{\pi}$ & $\chi_{v}$ & $c_{0}^{\pi}$ & $c_{2}^{\pi}$ \\
\hline${ }^{146} \mathrm{Nd}$ & 5 & 2 & 0.90 & -0.150 & -1.2 & 0.0 & 0.4 & 0.2 \\
${ }^{148} \mathrm{Nd}$ & 5 & 3 & 0.73 & -0.100 & -1.2 & -0.8 & 0.4 & 0.2 \\
${ }^{150} \mathrm{Nd}$ & 5 & 4 & 0.48 & -0.070 & -1.2 & -1.0 & 0.4 & 0.2 \\
${ }^{152} \mathrm{Nd}$ & 5 & 5 & 0.37 & -0.089 & -1.2 & -1.1 & 0.4 & 0.2 \\
${ }^{148} \mathrm{Sm}$ & 6 & 2 & 0.90 & -0.120 & -1.3 & 0.0 & 0.0 & 0.05 \\
${ }^{150} \mathrm{Sm}$ & 6 & 3 & 0.70 & -0.076 & -1.3 & -0.8 & 0.0 & 0.05 \\
${ }^{152} \mathrm{Sm}$ & 6 & 4 & 0.52 & -0.071 & -1.3 & -1.0 & 0.0 & 0.05 \\
${ }^{154} \mathrm{Sm}$ & 6 & 5 & 0.44 & -0.079 & -1.3 & -1.1 & 0.0 & 0.05 \\
${ }^{150} \mathrm{Gd}$ & 7 & 2 & 0.95 & -0.090 & -1.0 & 0.0 & -0.2 & -0.1 \\
${ }^{152} \mathrm{Gd}$ & 7 & 3 & 0.70 & -0.070 & -1.0 & -0.8 & -0.2 & -0.1 \\
${ }^{154} \mathrm{Gd}$ & 7 & 4 & 0.55 & -0.072 & -1.0 & -1.0 & -0.2 & -0.1 \\
${ }^{156} \mathrm{Gd}$ & 7 & 5 & 0.46 & -0.073 & -1.0 & -1.1 & -0.2 & -0.1 \\
\hline
\end{tabular}

Table 2

Strengths of the Bose-Fermi couplings in the odd-even nuclei ${ }^{147-153} \mathrm{Pm},{ }^{149-155} \mathrm{Eu},{ }^{151-157} \mathrm{~Tb}$.

\begin{tabular}{lllll}
\hline & $A_{s}$ & $2 \Gamma_{s}$ & $\Lambda_{s}$ & $v_{j}^{2}$ \\
\hline${ }^{147} \mathrm{Pm}$ & -0.1 & 0.94 & 2.52 & 0.18 \\
${ }^{149} \mathrm{Pm}$ & -0.1 & 1.26 & 3.39 & 0.25 \\
${ }^{151} \mathrm{Pm}$ & -0.1 & 1.52 & 4.11 & 0.33 \\
${ }^{153} \mathrm{Pm}$ & -0.1 & 1.67 & 4.51 & 0.33 \\
${ }^{149} \mathrm{Eu}$ & -0.1 & 0.83 & 1.91 & 0.30 \\
${ }^{151} \mathrm{Eu}$ & -0.1 & 1.15 & 2.63 & 0.32 \\
${ }^{153} \mathrm{Eu}$ & -0.1 & 1.36 & 3.45 & 0.34 \\
${ }^{155} \mathrm{Eu}$ & -0.1 & 1.51 & 3.53 & 0.35 \\
${ }^{151} \mathrm{~Tb}$ & -0.1 & 0.73 & 1.25 & 0.36 \\
${ }^{153} \mathrm{~Tb}$ & -0.1 & 1.05 & 1.97 & 0.38 \\
${ }^{155} \mathrm{~Tb}$ & -0.1 & 1.26 & 2.79 & 0.40 \\
${ }^{157} \mathrm{~Tb}$ & -0.1 & 1.41 & 2.87 & 0.41 \\
\hline
\end{tabular}




\subsection{Two-neutron separation energies}

A complete analysis of two-neutron separation energies requires a calculation of both positive and negative parity states in ${ }_{61} \mathrm{Pm},{ }_{63} \mathrm{Eu}$ and ${ }_{65} \mathrm{~Tb}$. For the Eu isotopes this calculation was done by Schol-

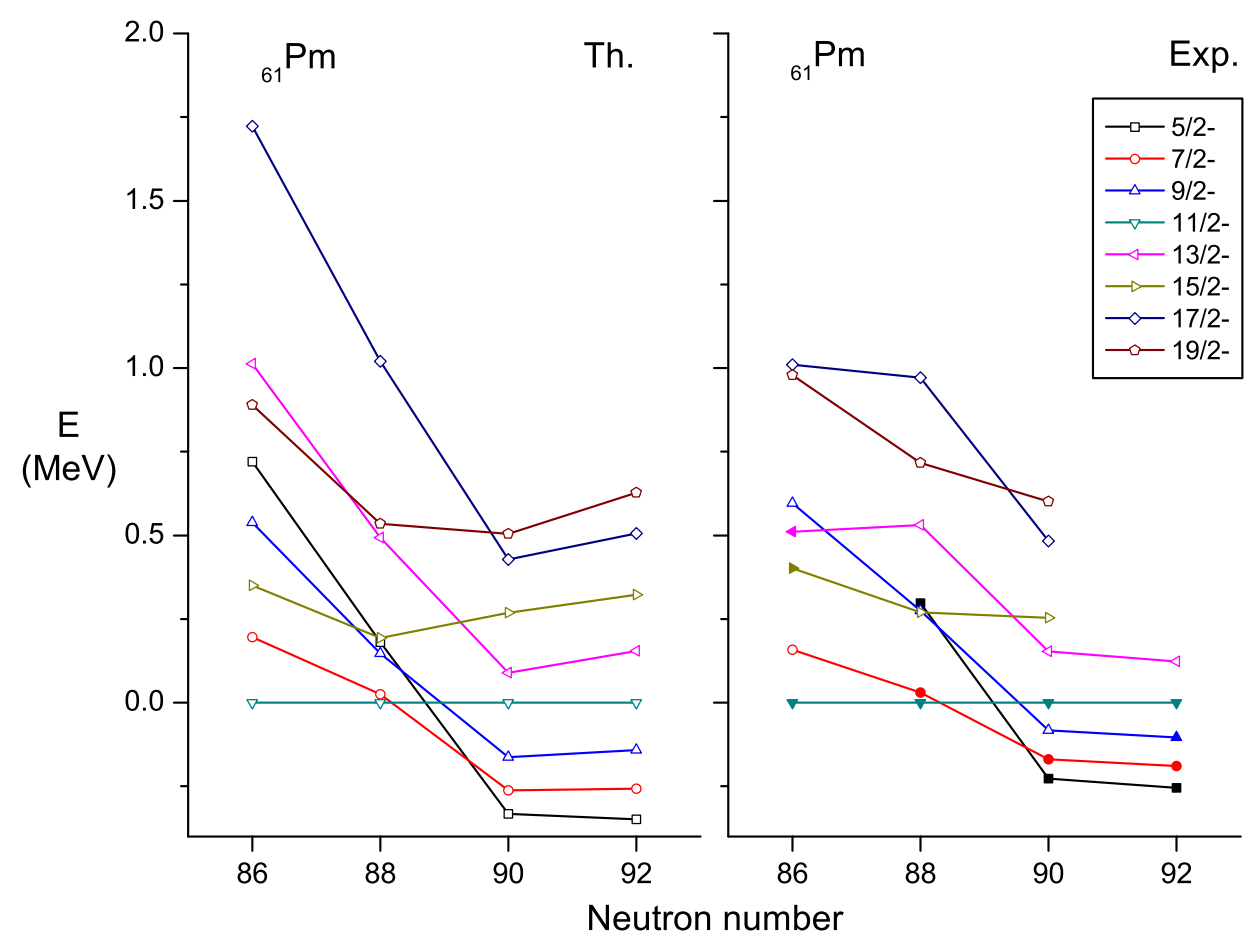

Fig. 24. Comparison between calculated and experimental spectra of negative parity states in ${ }_{61} \mathrm{Pm}$. The lowest $11 / 2^{-}$state is taken as zero of the energy. The parameters of the calculation are given in Tables 1 and 2. In the experimental spectra, taken from [45], uncertain assignments are indicated by open symbols.

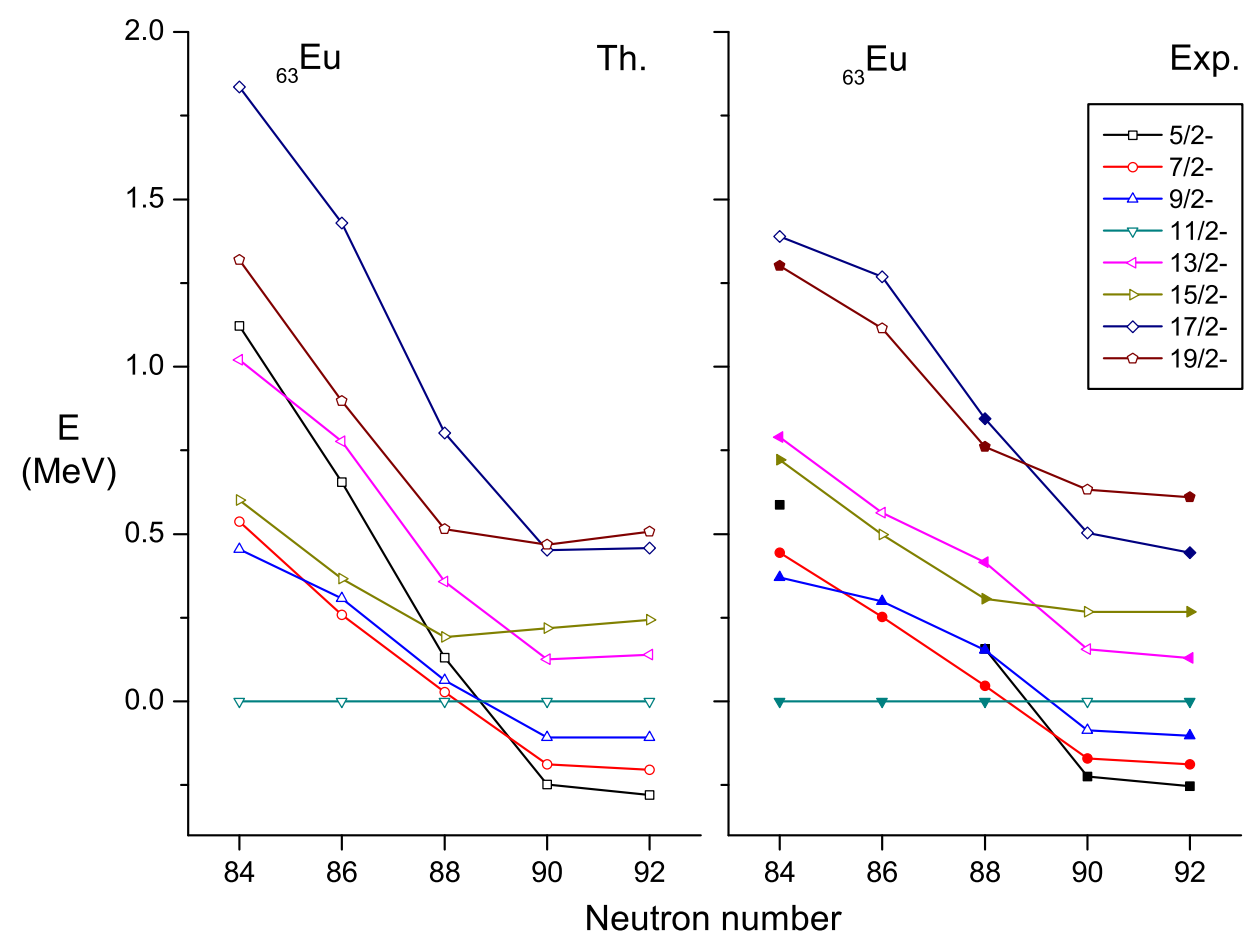

Fig. 25. Same as Fig. 24 but for ${ }_{63} \mathrm{Eu}$. 


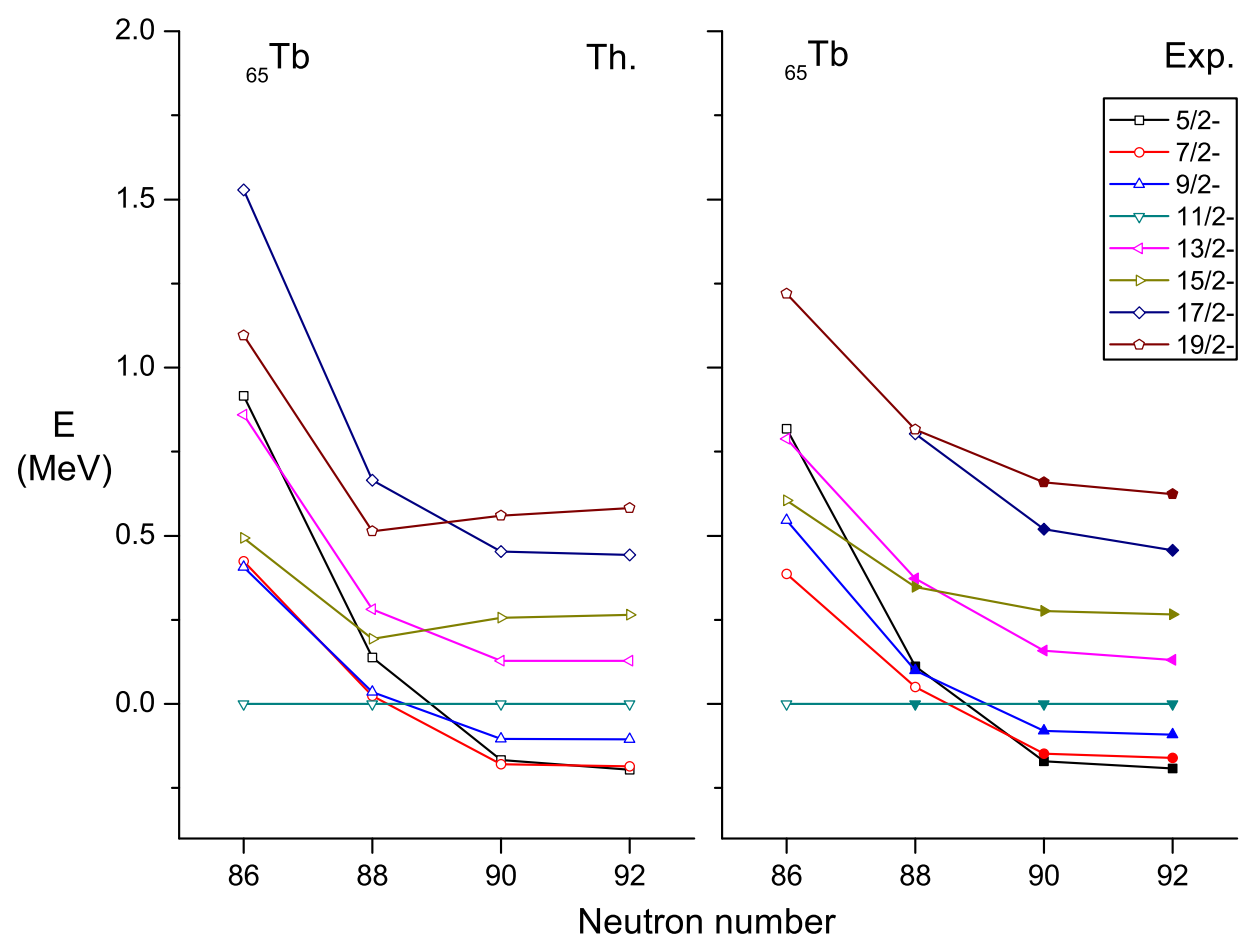

Fig. 26. Same as Fig. 25 but for ${ }_{65} \mathrm{~Tb}$.

ten [29]. The experimental two-neutron separation energies in ${ }_{61} \mathrm{Pm},{ }_{63} \mathrm{Eu}$ and ${ }_{65} \mathrm{~Tb}[46]$ are shown in Fig. 27.

One can see clearly the occurrence of discontinuities in the behavior of the two-neutron separation energies, an indication of a QPT. In order to emphasize these discontinuities, we note that the two neutron separation energies are given by a smooth contribution linear in $N$ plus the contribution of the deformation [7, p.74]

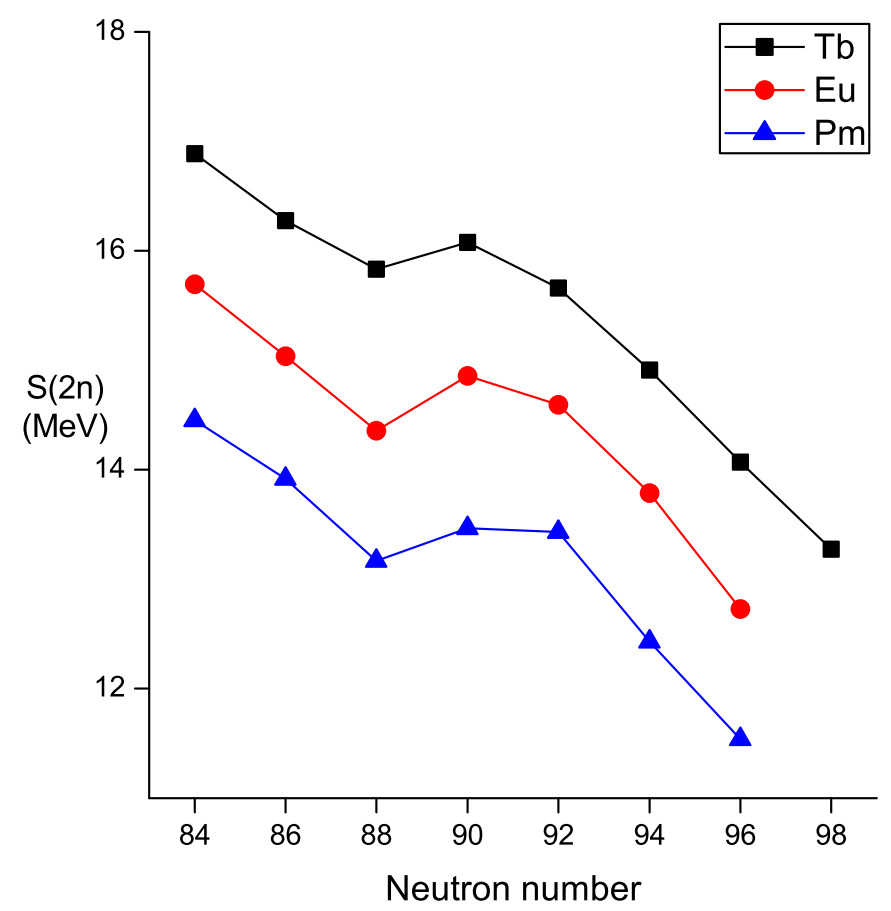

Fig. 27. Experimental two-neutron separation energies, $S(2 n)$, for the even-neutron isotopes of ${ }_{61} \mathrm{Pm},{ }_{63} \mathrm{Eu}$ and ${ }_{65} \mathrm{~Tb}$, taken from [46]. 


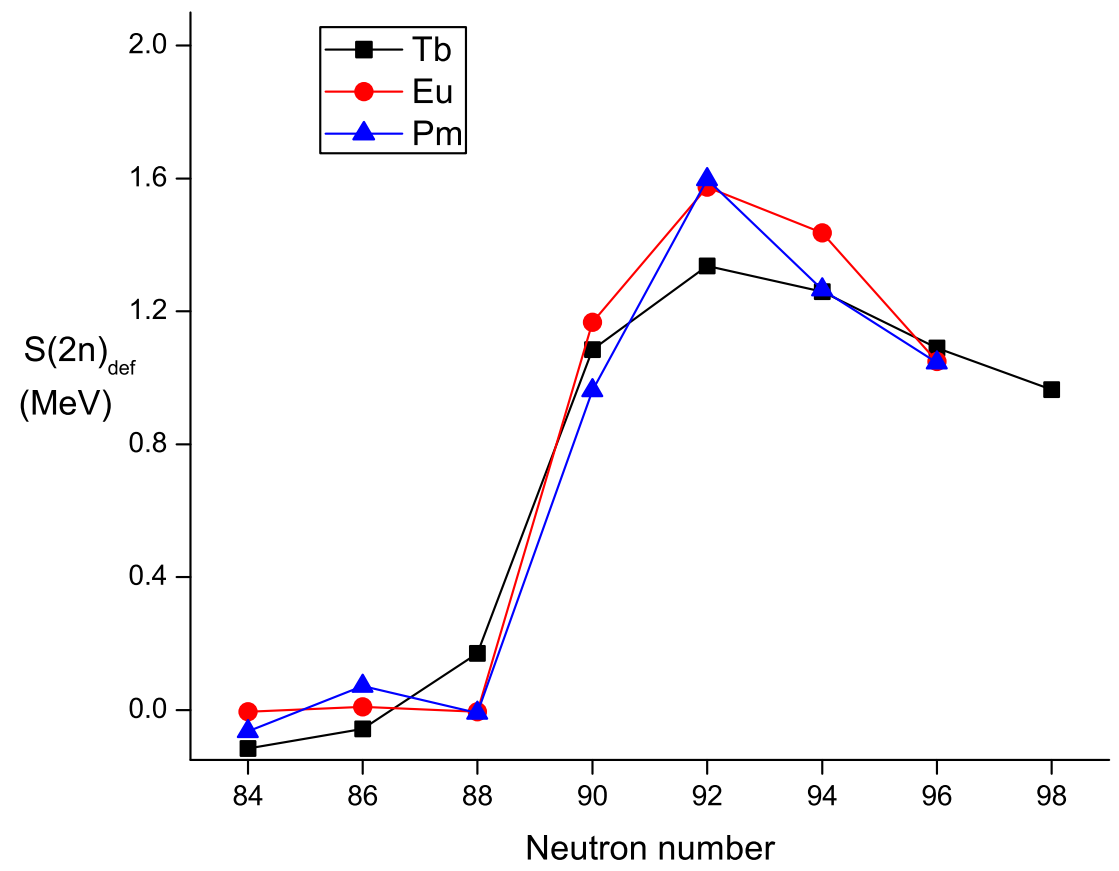

Fig. 28. The contribution of deformation, $S(2 n)_{d e f}$ to the two-neutron separation energies, Eq. (47), in ${ }_{61} \mathrm{Pm},{ }_{63} \mathrm{Eu}$ and ${ }_{65} \mathrm{~Tb}$. Experimental data taken from [46].

$$
S_{2 n}=-A_{2 n}-B_{2 n} N+S_{2 n}^{\text {def }}
$$

In Fig. 28 we show the deformation contribution, extracted from the data by subtracting the linear dependence, with $A_{2 n}=-15.185,-16.37,-17.672 \mathrm{MeV}$ for $\mathrm{Pm}, \mathrm{Eu}, \mathrm{Tb}$, respectively, and $B_{2 n}=0.670 \mathrm{MeV}$. The deformation contribution can be easily calculated using IBFM. Fig. 29 shows this contribution for the $h_{11 / 2}$ level, for ${ }_{61} \mathrm{Pm},{ }_{63} \mathrm{Eu}$ and ${ }_{65} \mathrm{~Tb}$. Figs. 28 and 29 cannot be, in principle, directly compared since the experimental data are the separation energies of the actual ground state, while Fig. 29 shows the separation energies of the $h_{11 / 2}$ level. However, in practice, differences between

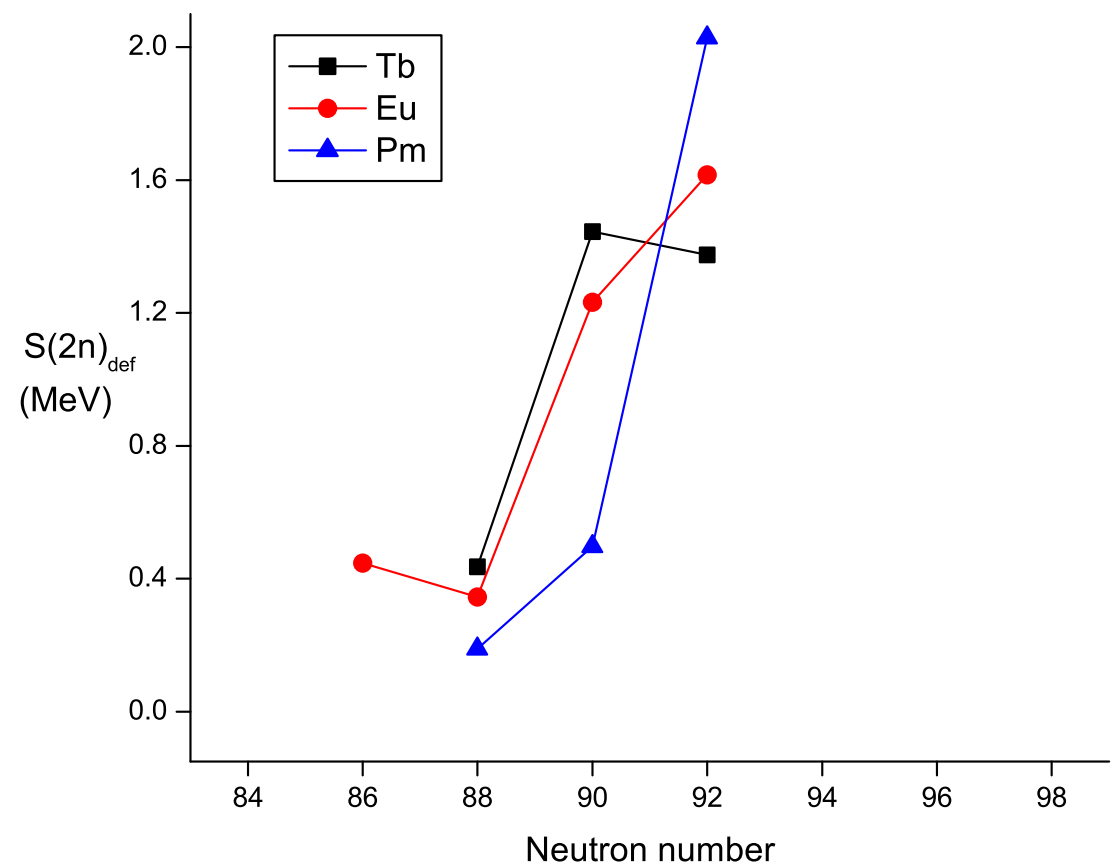

Fig. 29. The contribution of deformation, $S(2 n)_{d e f}$ Eq. (47), calculated in IBFM with the parameters given in the text. 
the two are of order $1 / N$, and thus, to a good approximation, Fig. 28 can be compared to Fig. 29. In particular, the onset of deformation is clearly seen both in Figs. 28 and 29.

\section{Conclusions}

In this paper, we have analyzed QPTs in Bose-Fermi systems, specifically the effect of one singlefermion with angular momentum $j$ immersed in a bath of bosons with angular momentum $L=0,2(s, d$ bosons).

By doing a classical analysis (Section 3) we have studied the single particle motion in a field with $\beta$, $\gamma$ deformation and determined the classical order parameters $\beta_{e, i}, \gamma_{e, i}$. We have shown that while the presence of the odd-fermion does not influence much the motion in the strongly deformed regions, the corrections being of order $1 / N$, it does influence greatly the location of the critical point and the entire nature of the phase transition, washing out the transition for some states and enhancing it for others. This is a novel result which may have applications to other fields of physics outside of nuclear physics.

By doing a quantal analysis (Section 4) we have investigated the change in level structure induced by the phase transition (correlation diagrams). Here we have obtained the result that while the presence of the odd-nucleon does not affect much the ground-state energy $(1 / N$ correction) it does affect greatly the level structure. This level structure is rather complex, especially in the neighborhood of the critical point. We have also used the correlation diagrams to investigate the classical-quantal correspondence and shown that when the bosons have SU(3) symmetry the correspondence is exact.

Finally, we have presented experimental evidence for the $\mathrm{U}(5)-\mathrm{SU}(3)$ transition (spherical to axially deformed) in odd-proton nuclei, ${ }_{61} \mathrm{Pm},{ }_{63} \mathrm{Eu}$ and ${ }_{65} \mathrm{~Tb}$, and performed a realistic calculation which accounts well for the experimental level structure. This is in spite of the fact that the structure of these nuclei is rather complex and cannot be simply described either by the rotational or by the vibrational model.

Our investigation of the effects of a single fermion on the phase transitions of a boson condensate has been done for the rather complex case of bosons with angular momentum $L=0,2$ and fermion with angular momentum $j=11 / 2$. It applies equally well to simpler cases, for example to the case of a spin $j=1 / 2$ particle immersed in a bath of spinless bosons $L=0$. As such, the method discussed here can be used in a variety of fields, ranging from molecules to atomic condensates, from nuclei to mesoscopic systems.

\section{Acknowledgements}

We thank C.E. Alonso, J.M. Arias, M. Böyükata, M.A. Caprio, L. Fortunato and especially, A. Vitturi for many useful discussions on their work in QPT in Bose-Fermi systems. This work was performed in part under DOE Grant No. DE-FG-02-91ER40608 and in part by a grant from the US-Israel Binational Science Foundation.

\section{References}

[1] R. Gilmore, D.H. Feng, Nucl. Phys. A 301 (1978) 189.

[2] R. Gilmore, J. Math. Phys. 20 (1979) 891.

[3] M. Vojta, Rep. Prog. Phys. 66 (2003) 2069.

[4] A.E.L. Dieperink, O. Scholten, F. Iachello, Phys. Rev. Lett. 44 (1980) 1747.

[5] D.H. Feng, R. Gilmore, S.R. Deans, Phys. Rev. C 23 (1981) 1254.

[6] O. Scholten, F. Iachello, A. Arima, Ann. Phys. (NY) 115 (1978) 325

[7] F. Iachello, A. Arima, The Interacting Boson Model, Cambridge University Press, Cambridge, 1987.

[8] F. Iachello, Phys. Rev. Lett. 85 (2000) 3580.

[9] F. Iachello, Phys. Rev. Lett. 87 (2001) 052502.

[10] F. Iachello, Phys. Rev. Lett. 91 (2003) 132502.

[11] D.J. Rowe, Phys. Rev. Lett. 93 (2004) 47.

[12] A. Leviatan, Phys. Rev. Lett. 98 (2007) 242502.

[13] R.F. Casten, E.M. McCutchan, J. Phys. G: Nucl. Part. Phys. 34 (2007) R285.

[14] R.F. Casten, Prog. Part. Nucl. Phys. 62 (2009) 183.

[15] A. Leviatan, J.N. Ginocchio, Phys. Rev. Lett. 90 (2003) 212501. 
[16] F. Iachello, N.V. Zamfir, Phys. Rev. Lett. 92 (2004) 212501.

[17] A. Leviatan, Phys. Rev. C 72 (2005) 031305(R).

[18] A. Leviatan, Phys. Rev. C 74 (2006) 051301(R).

[19] D.J. Rowe, P.S. Turner, G. Rosensteel, Phys. Rev. Lett. 93 (2004) 232502.

[20] S. Dusuel, J. Vidal, J.M. Arias, J. Dukelsky, J.E. Garcia-Ramos, Phys. Rev. C 72 (2005) 011301(R).

[21] E. Williams, A Study of Transitional Collective Behaviour in Heavy Nuclei, Ph.D. Thesis, Yale University, 2009.

[22] E. Williams, R.J. Casperson, V. Werner, Phys. Rev. C 81 (2010) 054306.

[23] M.A. Caprio, P. Cejnar, F. Iachello, Ann. Phys. (NY) 323 (2008) 1106.

[24] P. Cejnar, J. Jolie, Prog. Part. Nucl. Phys. 62 (2009) 210.

[25] P. Cejnar, J. Jolie, R.F. Casten, Rev. Mod. Phys. 82 (2010) 2155.

[26] F. Iachello, M.A. Caprio, in: L. Carr (Ed.), Understanding Quantum Phase Transitions, Taylor and Francis, 2010.

[27] F. Iachello, P. Van Isacker, The Interacting Boson-Fermion Model, Cambridge University Press, Cambridge, 1991.

[28] K. Le Hur, in: L. Carr (Ed.), Understanding Quantum Phase Transitions, Taylor and Francis, 2010.

[29] O. Scholten, N. Blasi, Nucl. Phys. A 380 (1982) 509.

[30] C.E. Alonso, J.M. Arias, L. Fortunato, A. Vitturi, Phys. Rev. C 72 (2005) 061302(R).

[31] C.E. Alonso, J.M. Arias, A. Vitturi, Phys. Rev. C 75 (2007) 064316.

[32] C.E. Alonso, J.M. Arias, L. Fortunato, A. Vitturi, Phys. Rev. C 79 (2009) 014306.

[33] M. Böyükata, C.E. Alonso, J.M. Arias, L. Fortunato, A. Vitturi, Phys. Rev. C 82 (2010) 014317.

[34] F. Iachello, in: A. Molinari, L. Riccati, W.M. Alberico, M. Morando (Eds.), Proceedings of the International School "Enrico Fermi", Course CLIII, IOS Press, Amsterdam, 2003.

[35] L. Landau, E.M. Lifshitz, Statistical Physics, Pergamon, Oxford, 1980.

[36] A. Leviatan, Phys. Lett. B 209 (1988) 415.

[37] Amiram Leviatan, Bin Shao, Phys. Rev. Lett. 63 (1989) 2204.

[38] J.N. Ginocchio, M.W. Kirson, Phys. Rev. Lett. 44 (1980) 1744

[39] A. Bohr, B.R. Mottelson, Phys. Scr. 22 (1980) 468.

[40] J. Meyer-ter-Vehn, Nucl. Phys. A 249 (1975) 111.

[41] S.G. Nilsson, K. Dan, Vidensk. Selsk. Mat.-Fys. Medd. 29 (16) (1955).

[42] O. Scholten, Computer Program ODDA.

[43] O. Scholten, The Interacting Boson Approximation Model and Applications, Ph.D. Thesis, University of Groningen, The Netherlands, 1980.

[44] O. Scholten, T. Ozzello, Nucl. Phys. A 424 (1984) 221.

[45] Evaluated Nuclear Structure Data File (ENSDF), <http://www.nndc.bnl.gov/ensdf/browse_top.jsp>.

[46] LBNL Isotopes Project Nuclear Data Dissemination Home Page, retrieved April 30, 2010, <http://ie.lbl.gov/toi2003/ MassSearch.asp>. 